\title{
Approximate radical for clusters: a global ap- proach using Gaussian elimination or SVD
}

\author{
Itnuit Janovitz-Freireich, Lajos Rónyai and Ágnes Szántó
}

\begin{abstract}
We present a method based on Dickson's lemma to compute the "approximate radical" of a zero dimensional ideal $\tilde{I}$ in $\mathbb{C}\left[x_{1}, \ldots, x_{m}\right]$ which has zero clusters: the approximate radical ideal has exactly one root in each cluster for sufficiently small clusters. Our method is "global" in the sense that it does not require any local approximation of the zero clusters: it reduces the problem to the computation of the numerical nullspace of the so called "matrix of traces", a matrix computable from the generating polynomials of $\tilde{I}$. To compute the numerical nullspace of the matrix of traces we propose to use Gauss elimination with pivoting or singular value decomposition. We prove that if $\tilde{I}$ has $k$ distinct zero clusters each of radius at most $\varepsilon$ in the $\infty$-norm, then $k$ steps of Gauss elimination on the matrix of traces yields a submatrix with all entries asymptotically equal to $\varepsilon^{2}$. We also show that the $(k+1)$-th singular value of the matrix of traces is proportional to $\varepsilon^{2}$. The resulting approximate radical has one root in each cluster with coordinates which are the arithmetic mean of the cluster, up to an error term asymptotically equal to $\varepsilon^{2}$. In the univariate case our method gives an alternative to known approximate square-free factorization algorithms which is simpler and its accuracy is better understood.
\end{abstract}

Mathematics Subject Classification (2000). Primary 65D20; Secondary 33F10. Keywords. radical ideal, clusters, matrix of traces, symbolic-numeric computation.

\section{Introduction}

Let $I \subset \mathbb{C}[\mathbf{x}]$ be a polynomial ideal in $m$ variables $\mathbf{x}=\left[x_{1}, \ldots, x_{m}\right]$ with roots $\mathbf{z}_{1}, \ldots, \mathbf{z}_{k} \in \mathbb{C}^{m}$ of multiplicities $n_{1}, \ldots, n_{k}$, respectively, and let $\tilde{I} \in \mathbb{C}[\mathbf{x}]$ be an ideal with clusters $C_{1}, \ldots, C_{k}$ such that each cluster $C_{i}$ has $n_{i}$ roots around $\mathbf{z}_{i}$

This work was completed with the support of NSF grants CCR-0306406 and CCR-0347506 and OTKA grants T42481 and T42706 and NK63066. 
within radius $\varepsilon$ in the $\infty$-norm for $i=1, \ldots, k$. We present an algorithm which computes an approximate radical of $\tilde{I}$, denoted by $\widetilde{\sqrt{I}}$, which has exactly one root for each cluster, and we show that such root corresponds to the arithmetic mean of the cluster.

The method we present in the paper is "global" in the sense that we do not use any local information about the roots in the clusters, only the coefficients of the system of polynomials defining $\tilde{I}$, and we return another system of polynomials where all near multiplicities are eliminated. In the univariate case such global algorithms are used for example in approximate factoring (see [25]), where the input polynomial needs to be "square-free" in the approximate sense. Previous global methods which handle univariate polynomials with clusters use approximate gcd computation and approximate polynomial division in order to either factor out the near multiplicities or to compute the approximate multiplicity structure and find the roots of the nearest polynomial with the given multiplicity structure [44, 21, 25, 51. The method we propose here offers an alternative algorithm to factor out near multiplicities, which is simpler, and the relation between the accuracy of the output and the size of the clusters is better understood. We describe separately our method applied to the univariate case, and illustrate its simplicity and accuracy.

Our method is based on Dickson's lemma, which gives the Jacobson radical of a finite dimensional associative algebra over a field of characteristic 0 via the vanishing of traces of elements in the algebra. An immediate application of Dickson's lemma to the algebra $\mathbb{C}[\mathbf{x}] / I$ finds a basis for $\sqrt{I} / I$ by finding the nullspace of the matrix of traces $R$, a matrix computable from the generating polynomials of $I$ using either multiplication matrices or other trace computation methods, as described below.

The main focus of the paper is to adapt the method based on Dickson's lemma to the case when the ideal $\tilde{I}$ has clusters of roots. In the paper we assume that both $\mathbb{C}[\mathbf{x}] / I$ and $\mathbb{C}[\mathbf{x}] / \tilde{I}$ are finite dimensional over $\mathbb{C}$ and have the same basis $B \subset \mathbb{C}[\mathbf{x}]$. Note that if $I$ is generated by a well-constrained system, then "almost all" perturbations $\tilde{I}$ of $I$ will satisfy our assumption, however our results are not limited to well-constrained systems only. On the other hand, the results we prove in this paper measure the accuracy of the output in terms of the size of the clusters, as opposed to the size of the perturbation of the generating polynomials of the ideal $I$. The extension of our method to handle perturbations which change the structure of the factor algebra and to understand the accuracy of the output in terms of the size of the coefficient perturbation is the subject of future research. The results in this paper can be summarized as follows:

Given the basis $B$ and the matrix of traces $R$ associated to $\tilde{I}$ and $B$, using Gaussian elimination with complete pivoting (GECP) we give asymptotic estimates of order $\varepsilon^{2}$ for the "almost vanishing" entries in $U_{k}$, the partially row reduced matrix of $R$, as well as upper bounds for the coefficients of $\varepsilon^{2}$, where $\varepsilon$ is the radius of the clusters in the $\infty$-norm. These bounds can be used to give a 
threshold to decide on the numerical rank of $R$, and to indicate the relationship between the numerical rank and the size of the clusters.

Alternatively, we show how our results for the GECP of the matrix of traces $R$ imply asymptotic bounds on the singular values of $R$. We also obtain in this case that the "almost vanishing" singular values are proportional to the square of the size of the clusters. This implies that for the numerical rank determination of $R$, computing its $S V D$ works similarly as using GECP.

Using a basis of the numerical nullspace of $R$ (or possibly an extended version of it), we define a set of generating polynomials for the approximate radical ideal $\widetilde{\sqrt{I}}$, or similarly, define a system of multiplication matrices $M_{x_{1}}^{\prime}, \ldots, M_{x_{m}}^{\prime}$ of $\mathbb{C}[\mathbf{x}] / \widetilde{\sqrt{I}}$ with respect to a basis $B^{\prime}$. We prove that modulo $\varepsilon^{2}$ the generating polynomials of $\widetilde{\sqrt{I}}$ are consistent and have roots with coordinates which are the arithmetic means of the coordinates of the roots in the clusters, which implies that the matrices $M_{x_{1}}^{\prime}, \ldots, M_{x_{m}}^{\prime}$ commute and their eigenvalues are the arithmetic means of the coordinates of the roots in the clusters, all modulo $\varepsilon^{2}$. In other words, our algorithm finds the coefficients of a polynomial system with roots which are the means of the clusters up to a precision of about twice as many digits as the radius of the clusters, assuming that the clusters are sufficiently small.

Let us briefly mention some of the possible methods to compute the matrix of traces $R$, although in the paper we do not elaborate on this aspect. As we shall demonstrate in the paper, the matrix of traces $R$ is readily computable from a system of multiplication matrices of $\mathbb{C}[\mathbf{x}] / \tilde{I}$, for example from $M_{x_{1}}, \ldots, M_{x_{m}}$, where $M_{x_{i}}$ denotes the matrix of the multiplication map by $x_{i}$ in $\mathbb{C}[\mathbf{x}] / \tilde{I}$ written in terms of the basis $B$. One can compute $M_{x_{i}}$ using Gröbner bases (see for example 9), resultant and subresultant matrices 30, 8, 49, Lazard's algorithm 27, 11, or by methods that combine these 38. Thus, our algorithm reduces the problem of finding the eigenvalues of matrices $M_{x_{1}}, \ldots, M_{x_{m}}$ which have clustered eigenvalues to finding eigenvalues of the smaller matrices $M_{x_{1}}^{\prime}, \ldots, M_{x_{m}}^{\prime}$ with well separated eigenvalues.

In certain cases, the matrix of traces can be computed directly from the generating polynomials of $\tilde{I}$, without using multiplication matrices. We refer to the papers [4, 17, 6, 7, 5, for the computation of traces using residues and Newton sums, or 13] using resultants.

Also, fast computation techniques like the "baby steps-giant steps" method 24, 46, 45, can be implemented to speed up the computation of all $n^{2}$ entries of the matrix of traces. As we prove in the paper, the entries of the matrix of traces $R$ are continuous in the size $\varepsilon$ of the root perturbation around $\varepsilon=0$, unlike the entries of multiplication matrices which may have many accumulation points as $\varepsilon$ approaches zero. Therefore, avoiding the computation of the multiplication matrices has the advantage of staying away from the possible large computational errors caused by the discontinuity of their entries. 
In the multivariate case, most of the methods handling clusters of roots in the literature are "local" in that they assume sufficiently close approximations for the clusters in question. Our algorithm, viewed as having the multiplication matrices as input, is closest to the approach in [30, 10, in that these papers also reduce the problem to the computation of the eigenvalues of a system of approximate multiplication matrices. Both of these papers propose to reorder the eigenvalues of the multiplication matrices to group the clusters together. For the reordering of the eigenvalues these papers compute approximations of the eigenvalues by either using the approach in [2] or using the univariate method of [21]. In contrast, our method reorders the eigenvalues of all multiplication matrices simultaneously without approximating the eigenvalues, grouping one eigenvalue from each of the clusters together in a way which facilitates the computation of the means of the clusters and the elimination of the rest of the nearly repeated eigenvalues. Another local method to handle near multiple roots is the "deflation" algorithm, studied in the works [41, 39, 40, 28, 29, to replace the original system which had a near multiple root with another one which has the same root with multiplicity one, using an approximation of the root in question. Related to the deflation algorithm, in 47, 48, 14 methods are proposed to compute the multiplicity structure of a root locally in terms of the so called dual basis, and then computing good approximations for the individual roots in the cluster, assuming that either a near system with multiple roots is known, or a sufficient approximation of the multiple root is given. Additionally, methods for computing singular solutions of both polynomials and analytic functions using homotopy continuation can be found in 33, 34, 35.

We also include here reference to some of the related methods for solving systems of polynomial equations with exact multiplicities: involving the computation of dual bases [32, 31, 48, or in the univariate (or bivariate) case, using Gauss maps 26, or analyzing the structure of the multiplication matrices by transforming them to an upper triangular form [50, 36, 37]. Previous work using Dickson's Lemma to compute radical ideals in the exact case includes [1, 3]. Also, 43] uses trace matrices in order to find separating linear forms deterministically.

The present paper is the extended and unabridged version of the paper that appeared in 22 .

\section{Acknowledgements:}

We would like to thank Erich Kaltofen for suggesting the problem.

\section{Preliminaries}

Let $A$ be an associative algebra over a field $F$ of characteristic 0. (See definition and basic properties of associative algebras in [19, 42].)

An element $x \in A$ is nilpotent if $x^{m}=0$ for some positive integer $m$.

An element $x \in A$ is properly nilpotent if $x y$ is nilpotent for every $y \in A$. 
The radical of $A$, denoted $\operatorname{Rad}(A)$, is the set of properly nilpotent elements of $A$. The radical $\operatorname{Rad}(A)$ is an ideal of $A$. In commutative algebras nilpotent elements are properly nilpotent, hence for a commutative $A$ the $\operatorname{radical} \operatorname{Rad}(A)$ is simply the set of nilpotent elements in $A$.

Throughout the paper we assume that $A$ is finite dimensional over $F$. Fix a basis $B=\left[b_{1}, \ldots, b_{n}\right]$ of $A$ (note that later we will need to fix the order of the elements in $B$, that is why we use vector notation). We call the multiplication matrix $M_{x}$ of $x \in A$ the matrix of the multiplication map

$$
\begin{aligned}
m_{x}: A & \longrightarrow A \\
{[g] } & \mapsto[x g]
\end{aligned}
$$

written in the basis $B$. It is easy to verify (cf. Page 8 in [42]) that the map $x \mapsto M_{x}$ is an algebra homomorphism, called regular representation from $A$ to $M_{n}(F)$.

The trace of $x$, denoted $\operatorname{Tr}(x)$, is the trace of the matrix $M_{x}$. It is independent of the choice of the basis.

\section{Matrix Traces and the Radical}

Our main construction is based on the following results describing the elements of the radical of an associative algebra $A$ using traces of elements:

Theorem 2.1 (Dickson [18] pp.106-107). An element $x$ of an associative algebra $A$ over a field $F$ of characteristic 0 is properly nilpotent if and only if $\operatorname{Tr}(x y)=0$ for every $y \in A$.

Corollary 2.2 (Friedl and Rónyai [19] p.156). Let $F$ be a field of characteristic 0 and $A$ a matrix algebra over $F$. Let $B=\left[b_{1}, \ldots, b_{n}\right]$ be a linear basis of $A$ over the field $F$. Then $x \in \operatorname{Rad}(A)$ if and only if $\operatorname{Tr}\left(x b_{i}\right)=0, \quad i=1, \ldots, n$.

We apply the above results to the special case of commutative algebras which are quotients of polynomial rings. Consider the system of polynomial equations

$$
\mathbf{f}(\mathbf{x})=0
$$

where $\mathbf{f}=\left\{f_{1}, \ldots, f_{l}\right\}$ and each $f_{i}$ is a polynomial in the variables $\mathbf{x}=\left[x_{1}, \ldots, x_{m}\right]$. Assume that the polynomials $f_{1}, \ldots, f_{l}$ have finitely many roots in $\mathbb{C}^{m}$, which implies that the algebra $A=\mathbb{C}[\mathbf{x}] / I$ is finite dimensional, where $I$ is the ideal generated by the polynomials in $\mathbf{f}$. Denote the dimension of $A$ over $\mathbb{C}$ by $n$ and let $B=\left[b_{1}, \ldots, b_{n}\right]$ be a basis of $A$. By slight abuse of notation we denote the elements of the basis $B$ which are in $A$ and some fixed preimages of them in $\mathbb{C}\left[x_{1}, \ldots, x_{m}\right]$ both by $b_{1}, \ldots, b_{n}$. Let $\left\{\mathbf{z}_{1}, \ldots, \mathbf{z}_{n}\right\} \subset \mathbb{C}^{m}$ be the set of common roots (not necessarily all distinct) of the polynomials in $\mathbf{f}$. Using the multiplication matrices $M_{f}$ associated to the elements $f \in A$ and the fact that

$$
\operatorname{Rad}(A)=\sqrt{I} / I \subseteq \mathbb{C}[\mathbf{x}] / I=A,
$$

we can reword Corollary 2.2 in the following way: 
Corollary 2.3. Let $p \in \mathbb{C}[\mathbf{x}]$ and $\bar{p}$ be the image of $p$ in $A$. Using the above notation, the following statements are equivalent:

(i) $p \in \sqrt{I}$

(ii) $\bar{p} \in \operatorname{Rad}(A)$

(iii) $\operatorname{Tr}\left(M_{\bar{p} b_{j}}\right)=0$ for all $j=1, \ldots, n$.

We can now use the previous corollary to characterize the radical of $A$ as the nullspace of a matrix defined as follows:

Definition 2.4. The matrix of traces is the $n \times n$ symmetric matrix:

$$
R=\left[\operatorname{Tr}\left(M_{b_{i} b_{j}}\right)\right]_{i, j=1}^{n}
$$

where $M_{b_{i} b_{j}}$ is the multiplication matrix of $b_{i} b_{j}$ as an element in $A$ in terms of the basis $B=\left[b_{1}, \ldots, b_{n}\right]$ and $\operatorname{Tr}$ indicates the trace of a matrix.

Corollary 2.5. An element

$$
r=\sum_{k=1}^{n} c_{k} b_{k}
$$

of the quotient ring $A$ with basis $B=\left[b_{1}, \ldots, b_{n}\right]$ is in the radical of $A$ if and only if $\left[c_{1}, \ldots, c_{n}\right]$ is in the nullspace of the matrix of traces $R$.

Proof. Corollary 2.3 states that an element $r=\sum_{k=1}^{n} c_{k} b_{k} \in A$ belongs to $\operatorname{Rad}(A)$ if and only if $\operatorname{Tr}\left(M_{r b_{j}}\right)=0$, for all $j=1, \ldots, n$. From the linearity of both the multiplication map (see Proposition (4.2) in Chapter 2 of [12]) and the traces of matrices we have that

$$
\begin{aligned}
\operatorname{Tr}\left(M_{r b_{j}}\right) & =\sum_{k=1}^{n} c_{k} \operatorname{Tr}\left(M_{b_{k} b_{j}}\right) \\
& =\left[c_{1}, \ldots, c_{n}\right] R[j]
\end{aligned}
$$

where $R[j]$ is the $j^{t h}$ column of the matrix of traces $R$. Therefore, $\operatorname{Tr}\left(M_{r b_{j}}\right)=0$ for all $j=1, \ldots, n$ is equivalent to $\left[c_{1}, \ldots, c_{n}\right] R=0$.

Remark 2.6. Methods in the literature for computing the matrix of traces $R$ are mentioned in the Introduction. One way to compute it is from the multiplication matrices $M_{b_{i} b_{j}}$. Note that in order to compute the matrices $M_{b_{i} b_{j}}, i, j=1, \ldots, n$, it is sufficient to have $M_{x_{k}}, k=1, \ldots, m$, since if $h \in \mathbb{C}\left[x_{1}, \ldots, x_{m}\right]$ is a preimage of $b_{i} b_{j} \in A$, then we have

$$
\begin{aligned}
M_{b_{i} b_{j}} & =M_{h\left(x_{1}, \ldots, x_{m}\right)} \\
& =h\left(M_{x_{1}}, \ldots, M_{x_{m}}\right) .
\end{aligned}
$$

This is because the regular representation is a homomorphism of $\mathbb{C}$-algebras, see also Corollary (4.3) in Chapter 2 of [12. 
Example 2.7. We consider the polynomial system $f_{1}=f_{2}=f_{3}=0$, with

$$
\begin{aligned}
f_{1}= & x_{1}^{2}+4 x_{1} x_{2}-6 x_{1}+6 x_{2}^{2}-18 x_{2}+13 \\
f_{2}= & x_{1}^{3}+16 x_{1}^{2} x_{2}-7 x_{1}^{2}+118 x_{1} x_{2}^{2}-286 x_{1} x_{2} \\
& +147 x_{1}-x_{2}^{3}+6 x_{2}^{2}+x_{2}+5 \\
f_{3}= & x_{1}^{3}+10 x_{1}^{2} x_{2}-5 x_{1}^{2}+72 x_{1} x_{2}^{2}-176 x_{1} x_{2} \\
& +91 x_{1}-x_{2}^{3}+4 x_{2}^{2}+x_{2}+3
\end{aligned}
$$

These polynomials have two common roots: $[1,1]$ of multiplicity 3 and $[-1,2]$ of multiplicity 2.

We compute the multiplication matrices $M_{x_{1}}$ and $M_{x_{2}}$ with respect to the basis $B=\left[1, x_{1}, x_{2}, x_{1} x_{2}, x_{1}^{2}\right]$, which are respectively

$$
\left[\begin{array}{rrrrr}
0 & 1 & 0 & 0 & 0 \\
0 & 0 & 0 & 0 & 1 \\
0 & 0 & 0 & 1 & 0 \\
\frac{5}{3} & -2 & -1 & \frac{2}{3} & \frac{5}{3} \\
-\frac{17}{3} & 1 & 4 & \frac{4}{3} & \frac{1}{3}
\end{array}\right],\left[\begin{array}{rrrrr}
0 & 0 & 1 & 0 & 0 \\
0 & 0 & 0 & 1 & 0 \\
-\frac{13}{6} & 1 & 3 & -\frac{2}{3} & -\frac{1}{6} \\
-\frac{1}{6} & -1 & 0 & \frac{7}{3} & -\frac{1}{6} \\
\frac{5}{3} & -2 & -1 & \frac{2}{3} & \frac{5}{3}
\end{array}\right] .
$$

Here we used Chardin's subresultant construction to compute the multiplication matrices. (See 8, and 49.)

We now compute the matrix $R$ using Definition 2.4 and Remark 2.6;

$$
R=\left[\begin{array}{rrrrr}
5 & 1 & 7 & -1 & 5 \\
1 & 5 & -1 & 7 & 1 \\
7 & -1 & 11 & -5 & 7 \\
-1 & 7 & -5 & 11 & -1 \\
5 & 1 & 7 & -1 & 5
\end{array}\right]
$$

The nullspace of $R$ is generated by the vectors

$$
[1,-3,0,2,0],[0,-4,1,3,0],[0,-3,0,2,1] \text {. }
$$

By Corollary 2.5 we have that the radical of $I=\left\langle f_{1}, f_{2}, f_{3}\right\rangle$ modulo $I$ is

$$
\sqrt{I} / I=\left\langle 1-3 x_{1}+2 x_{1} x_{2},-4 x_{1}+x_{2}+3 x_{1} x_{2},-3 x_{1}+2 x_{1} x_{2}+x_{1}^{2}\right\rangle .
$$

Note that the polynomials on the right hand side are in $\sqrt{I}$.

Assume that rank $R=k$. Once we know the $n-k$ generators $\left\{r_{k+1}, \ldots, r_{n}\right\}$ of the radical, we can obtain the multiplication matrices of the elements of $A / \operatorname{Rad}(A)=$ $\mathbb{C}[\mathbf{x}] / \sqrt{I}$ by performing a change of basis on the multiplication matrices $M_{x_{1}}, \ldots, M_{x_{m}}$ to the basis $\left\{r_{1}, \ldots, r_{k}, r_{k+1}, \ldots, r_{n}\right\}$ of $A$, where $r_{1}, \ldots, r_{k}$ can be chosen arbitrarily as long as $\left\{r_{1}, \ldots, r_{k}, r_{k+1}, \ldots, r_{n}\right\}$ is linearly independent. Let $M_{x_{s}}$ be the multiplication matrix of the coordinate $x_{s}$ in the basis $\left[r_{1}, \ldots, r_{n}\right]$. Then the $k \times k$ principal submatrix

$$
M_{x_{s}}^{\prime}:=\left[M_{x_{s}}(i, j)\right]_{i, j=1}^{k}
$$

is the multiplication matrix of $x_{s}$ in $A / \operatorname{Rad}(A)=\mathbb{C}[\mathbf{x}] / \sqrt{I}$ with respect to the basis $\left[r_{1}, \ldots, r_{k}\right]$. 
Example 2.8. Continuing Example 2.7, we have that the generators of the radical $\operatorname{Rad}(A)$ have coordinates

$$
r_{3}=[1,-3,0,2,0], r_{4}=[0,-4,1,3,0], r_{5}=[0,-3,0,2,1]
$$

in the basis $B=\left[1, x_{1}, x_{2}, x_{1} x_{2}, x_{1}^{2}\right]$.

We set

$$
r_{1}=[1,0,0,0,0], r_{2}=[0,1,0,0,0] .
$$

We perform the change of basis to the two multiplication matrices $M_{x_{1}}$ and $M_{x_{2}}$ and obtain:

$$
\left[\begin{array}{rrrrr}
0 & 1 & 0 & 0 & 0 \\
1 & 0 & -1 & 0 & 1 \\
0 & 0 & 10 / 3 & -2 & 1 / 3 \\
0 & 0 & 5 & -3 & 1 \\
0 & 0 & -7 / 3 & 2 & 2 / 3
\end{array}\right] \text { and }\left[\begin{array}{rrrrr}
3 / 2 & -1 / 2 & -3 / 2 & 1 & 0 \\
-1 / 2 & 3 / 2 & 1 / 2 & 0 & 0 \\
0 & 0 & -1 / 3 & 1 & -1 / 3 \\
0 & 0 & -8 / 3 & 3 & -2 / 3 \\
0 & 0 & 4 / 3 & -1 & 4 / 3
\end{array}\right]
$$

respectively.

We then have that the multiplication matrices for $x_{1}$ and $x_{2}$ in $A / \operatorname{Rad}(A)$ in the basis $\left[1, x_{1}\right]$ are

$$
\mathcal{M}_{x_{1}}=\left[\begin{array}{ll}
0 & 1 \\
1 & 0
\end{array}\right] \text { and } \mathcal{M}_{x_{2}}=\left[\begin{array}{rr}
3 / 2 & -1 / 2 \\
-1 / 2 & 3 / 2
\end{array}\right] .
$$

The eigenvalues of these matrices give the solutions to the system.

\section{Clustered roots}

In this section we consider systems with clustered roots instead of systems with root multiplicities. We can think of these systems with clustered roots as being obtained from systems with multiplicities via one of the following two ways:

1. by perturbing the coefficients of the system with multiple roots,

2. by perturbing the multiple roots to obtain clusters.

Let $\mathbf{f}$ be the system with multiple roots and $\tilde{\mathbf{f}}$ be the system with clustered roots obtained from $\mathbf{f}$ by any of the above methods. Denote by $\tilde{A}=\mathbb{C}[\mathbf{x}] / \tilde{I}$ the algebra corresponding to the ideal $\tilde{I}$ generated by the polynomials in $\tilde{\mathbf{f}}$.

Assumption 3.1. Throughout this paper we make the assumption that the basis $B$ for $A$ also forms a basis for $\tilde{A}$. Note that if $\mathbf{f}$ is a well constrained system then for "almost all" perturbations $\tilde{\mathbf{f}}$ our assumption is satisfied, i.e. the set of perturbed systems for which it doesn't hold has measure zero in the space of all systems of given degrees.

If we assume that the basis $B$ for $A$ also forms a basis for $\tilde{A}$ then both the multiplication matrices and the matrix of traces are continuous functions of the coefficients of the polynomials. Therefore, small perturbations in the coefficients of $\mathbf{f}$ will result in small changes in the entries of the multiplication matrices and the matrix of traces. 
However, in case 2, when the roots are perturbed, the polynomials corresponding to the clustered system might end up having coefficients very different to those of the original system, even if the radii of the clusters were small. In this case, if we compute the multiplication matrices for the clustered system, the entries might not be continuous functions of the perturbation of the roots. They not only depend on the magnitude of the perturbation of the roots but also on the direction of the perturbation. However, as we shall show in Proposition 3.4 , the matrix of traces is always continuous in the roots. The following examples illustrates this phenomenon.

Example 3.2. We consider three examples of a single cluster of size proportional to $\varepsilon$ around the origin $(0,0)$ in $\mathbb{C}^{2}$ consisting of three roots. The first two examples demonstrate that the defining equations and the multiplication matrices can have different accumulation points as $\varepsilon$ approaches 0 , depending on the direction. The third example demonstrate that generally the defining equations and the multiplication matrices are not continuous at $\varepsilon=0$.

- First, the roots of the cluster are $(0,0),(\varepsilon, \varepsilon),(2 \varepsilon, 2 \varepsilon)$. The defining equations of these points in $\mathbb{C}[x, y]$ are given by $x^{3}-3 \varepsilon x^{2}+2 \varepsilon^{2} x=0$ and $y=x$, and the multiplication matrices in the basis $B=\left\{1, x, x^{2}\right\}$ are given by

$$
M_{x}=M_{y}=\left[\begin{array}{rrr}
0 & 1 & 0 \\
0 & 0 & 1 \\
0 & -2 \varepsilon^{2} & 3 \varepsilon
\end{array}\right] \quad \text { and } \quad \lim _{\varepsilon \rightarrow 0} M_{y}=\left[\begin{array}{lll}
0 & 1 & 0 \\
0 & 0 & 1 \\
0 & 0 & 0
\end{array}\right] \text {, }
$$

and the primary ideal defining the multiple root is $\left\langle x^{3}, x-y\right\rangle$.

- The next example has cluster $(0,0),(\varepsilon, 2 \varepsilon),(2 \varepsilon, 4 \varepsilon)$. The defining equations are $x^{3}-3 \varepsilon x^{2}+2 \varepsilon^{2} x=0$ and $y=2 x$. Then $M_{x}$ is the same as above, but

$$
M_{y}=\left[\begin{array}{rrr}
0 & 2 & 0 \\
0 & 0 & 2 \\
0 & -4 \varepsilon^{2} & 6 \varepsilon
\end{array}\right] \quad \text { and } \quad \lim _{\varepsilon \rightarrow 0} M_{y}=\left[\begin{array}{lll}
0 & 2 & 0 \\
0 & 0 & 2 \\
0 & 0 & 0
\end{array}\right] \text {, }
$$

and the primary ideal defining the multiple root is $\left\langle x^{3}, x-2 y\right\rangle$.

- More generally, the third example has cluster $(0,0),(\varepsilon, c \varepsilon),(2 \varepsilon, d \varepsilon)$ for some $c, d \in \mathbb{R}$. Then the first defining equation is the same as above, and the second equation is $y=-\frac{2 c-d}{2 \varepsilon} x^{2}+\left(2 c-\frac{d}{2}\right) x$ which is not continuous in $\varepsilon=0$, unless $d=2 c$. Similarly for the multiplication matrix $M_{y}$. However, the matrix of traces

$$
R=\left[\begin{array}{rrr}
3 & c \varepsilon+d \varepsilon & c^{2} \varepsilon^{2}+d^{2} \varepsilon^{2} \\
c \varepsilon+d \varepsilon & c^{2} \varepsilon^{2}+d^{2} \varepsilon^{2} & c^{3} \varepsilon^{3}+d^{3} \varepsilon^{3} \\
c^{2} \varepsilon^{2}+d^{2} \varepsilon^{2} & c^{3} \varepsilon^{3}+d^{3} \varepsilon^{3} & c^{4} \varepsilon^{4}+d^{4} \varepsilon^{4}
\end{array}\right]
$$

(with respect to the basis $\left\{1, y, y^{2}\right\}$ ) is continuous in $\varepsilon=0$ and has the same limit for every choices of $c$ and $d$.

Example 3.3. Continuing with Example 2.8, suppose now that instead of having a system with common roots $[1,1]$ of multiplicity 3 and $[-1,2]$ of multiplicity 2 we 
have a polynomial system with a cluster of three common roots:

$$
[[1,1],[0.9924,1.0027],[1.0076,0.9973]]
$$

around $[1,1]$ and a cluster of two common roots:

$$
[[-1,2],[-1.0076,2.0027]]
$$

around $[-1,2]$.

Using the multivariate Vandermonde construction (see for example [32]), we obtained the following multiplication matrices for this system, with respect to the same basis as for the system with multiple roots: $B=\left[1, x_{1}, x_{2}, x_{1} x_{2}, x_{1}^{2}\right]$.

$$
\begin{array}{r}
\tilde{M}_{x_{1}}=\left[\begin{array}{rrrrr}
3.8328 \times 10^{-6} & 9.9997 \times 10^{-1} & 3.0830 \times 10^{-8} & 4.1421 \times 10^{-7} & 3.9951 \times 10^{-5} \\
3.7919 \times 10^{-6} & -2.7338 \times 10^{-5} & 1.2303 \times 10^{-7} & 8.2891 \times 10^{-7} & 1.00004 \\
3.8527 \times 10^{-6} & -2.7463 \times 10^{-5} & -1.5183 \times 10^{-8} & 1.00000 & 3.9969 \times 10^{-5} \\
7.73947 & 21.69983 & -5.97279 & -16.79084 & -5.67565 \\
-17.94136 & -54.43008 & 13.97610 & 41.61207 & 17.78328
\end{array}\right] \\
\tilde{M}_{x_{2}}=\left[\begin{array}{rrrrr}
3.7831 \times 10^{-6} & -2.7103 \times 10^{-5} & & & \\
3.8527 \times 10^{-6} & -2.7464 \times 10^{-5} & -1.5183 \times 10^{-8} & -1.4715 \times 10^{-7} & 4.0017 \times 10^{-5} \\
-2.22905 & 1.06576 & 3.00000 & -7.1053 \times 10^{-1} & -1.2617 \times 10^{-1} \\
-3.23468 & -10.77768 & 2.47988 & 9.67839 & 2.85410 \\
7.73947 & 21.69983 & -5.97279 & -16.79084 & -5.67565
\end{array}\right]
\end{array}
$$

The norm of the difference between these matrices and the multiplication matrices (11) for the system with multiple roots are very large: 135.41 for the matrices of $x_{1}$ and 59.54 for the matrices of $x_{2}$. Entrywise, the largest absolute value of the difference of the entries of the matrices is 55.40 for $x_{1}$ and 21.70 for $x_{2}$.

However, the matrix of traces associated to the system with clusters is

$$
\left[\begin{array}{rrrrr}
4.99999 & 0.99240 & 7.00269 & -1.01796 & 5.01538 \\
0.99259 & 5.01557 & -1.01777 & 7.03349 & 0.97757 \\
7.00131 & -1.01934 & 11.00943 & -5.04274 & 7.03192 \\
-1.01900 & 7.03226 & -5.04240 & 11.07093 & -1.04951 \\
5.01548 & 0.97748 & 7.03339 & -1.04838 & 5.03155
\end{array}\right]
$$

and the 2-norm of the difference between this matrix and the multiplication matrix $R$ in (2) for the system with multiple roots is 0.147 .

We have the following result for the entries of the matrix of traces $R$ expressed in terms of the roots of the polynomial system.

Proposition 3.4. The matrix of traces $R$ of the system $\mathbf{f}(\mathbf{x})=0$ with respect to $B=\left[b_{1}, \ldots, b_{n}\right]$ can be expressed in terms of the common roots $\left\{\mathbf{z}_{1}, \ldots, \mathbf{z}_{n}\right\}$ as

$$
R=\left[\sum_{k=1}^{n} b_{i} b_{j}\left(\mathbf{z}_{k}\right)\right]_{i, j=1}^{n}
$$

where $b_{i} b_{j}\left(\mathbf{z}_{k}\right)$ indicates the evaluation of the polynomial $b_{i} b_{j}$ at the point $\mathbf{z}_{k}$.

Proof. Assume that $\left\{\mathbf{z}_{1}, \ldots, \mathbf{z}_{k}\right\}$ are the distinct elements among $\left\{\mathbf{z}_{1}, \ldots, \mathbf{z}_{n}\right\}$ in $V(I)$ and let $n_{i}$ be the multiplicity of $\mathbf{z}_{i}$. Let $Q_{i}$ be the (unique) primary component of $I$ in $\mathbb{C}[\mathbf{x}]$ whose radical $P_{i}$ is the ideal of all polynomials vanishing at $\mathbf{z}_{i}, i=$ $1,2, \ldots, k$. Set $A_{i}=\mathbb{C}[\mathbf{x}] / Q_{i}$. We have then $n_{i}=\operatorname{dim}_{\mathbb{C}} A_{i}$ and $I=Q_{1} \cap Q_{2} \cap \cdots Q_{k}$. 
Also the ideals $Q_{i}$ are pairwise relatively prime, hence by the Chinese Remainder Theorem we have

$$
A \cong A_{1} \oplus A_{2} \oplus \cdots \oplus A_{k}
$$

We denote also by $A_{i}$ the image of $A_{i}$ in $A$ at this isomorphism. Given any polynomial $g$, it is immediate that $A_{i}$ is an invariant subspace of the multiplication map $M_{g}$ and that the characteristic polynomial of $M_{g}$ on $A_{i}$ is $\left(t-g\left(\mathbf{z}_{i}\right)\right)^{n_{i}}$. This implies that the characteristic polynomial of $M_{g}$ is $\prod_{i=1}^{k}\left(t-g\left(\mathbf{z}_{i}\right)\right)^{n_{i}}=\prod_{i=1}^{n}\left(t-g\left(\mathbf{z}_{i}\right)\right)$. So the trace of $M_{g}$ is $\sum_{i=1}^{n} g\left(\mathbf{z}_{i}\right)$. Therefore

$$
\operatorname{Tr}\left(M_{b_{i} b_{j}}\right)=\sum_{k=1}^{n} b_{i} b_{j}\left(\mathbf{z}_{k}\right)
$$

which proves the lemma.

Note: An alternative proof can be given for Proposition 3.4 using the fact that the multiplication matrix $M_{g}$ is similar to a block diagonal matrix where the $i$-th diagonal block is an $n_{i} \times n_{i}$ upper triangular matrix, with diagonal entries $g\left(\mathbf{z}_{i}\right), i=1, \ldots, k$ (cf. [32, Theorem 2]).

The previous result shows that the entries of the matrix of traces are continuous functions of the roots, even when the roots coincide. In particular, a system with multiple roots and a system with clusters obtained by perturbing the roots of a system with multiplicities will have comparable matrices of traces.

\section{Univariate Case}

Before we give our method in full generality we would like to describe our algorithm in the univariate case. The purpose of this section is to demonstrate the simplicity and the accuracy of our technique to compute the approximate squarefree factorization of a univariate polynomial. As we mentioned in the Introduction, our method offers a new alternative to other approximate square-free factorization algorithms, such as the one in 25 .

The following is a description of the steps of our algorithm. Let

$$
f(x)=x^{d}+a_{1} x^{d-1}+\cdots+a_{d-1} x+a_{d} \in \mathbb{C}[x]
$$

be a given polynomial of degree $d$ with clusters of roots of size at most $\varepsilon$. The output of our algorithm is a polynomial $g(x) \in \mathbb{C}[x]$ such that its roots are the arithmetic means of the roots in each cluster, with a precision of order of magnitude $\varepsilon^{2}$.

1. Compute the matrix of traces $R$ w.r.t. the basis $B=\left[1, x, x^{2}, \ldots, x^{d-1}\right]$ using the Newton-Girard formulas. In this case we have $R=\left[s_{i+j}\right]_{i, j=0}^{d-1}$ where $s_{t}$ is the sum of the $t$-th power of the roots of $f$. We set $s_{0}=d$ and we find 
$s_{1}, \ldots, s_{2 d-2}$ from the coefficients of $f$ using the Newton-Girard formulas as follows:

$$
\begin{aligned}
s_{1}+a_{1} & =0 \\
s_{2}+a_{1} s_{1}+2 a_{2} & =0 \\
\vdots & \\
s_{d}+a_{1} s_{d-1}+\cdots+a_{d-1} s_{1}+d a_{d} & =0 \\
s_{d+1}+a_{1} s_{d}+\cdots+a_{d} s_{1} & =0 \\
\vdots & \\
s_{2 d-2}+a_{1} s_{2 d-3}+\cdots+a_{d} s_{d-3} & =0 .
\end{aligned}
$$

2. Gaussian elimination with complete pivoting (GECP) is used on the matrix $R$ until the remaining entries in the partially row reduced matrix $U_{k}$ are smaller than a preset threshold (see Propositions 5.7 and 5.8). The number of iterations performed, $k$, is the numerical rank of the matrix $R$.

3. Compute a basis of the nullspace $N$ of the first $k$ rows of the matrix $U_{k}$ obtained after $k$ steps of the GECP. We identify the vectors in $N$ by polynomials, by combining their coordinates with the corresponding basis elements of $B$.

4. The smallest degree polynomial in $N$ is the approximate square-free factor $g(x)$ of $f(x)$. Its roots are the arithmetic means of the roots in each cluster modulo $\varepsilon^{2}$ (see Proposition 7.5 ). In the case when the matrix $R$ has numerical rank $d$ then we take $g(x)=f(x)$ as the square-free factor.

Example 4.1. (1) Consider the approximate polynomial

$$
f=\left(x-\left(z+\delta_{1} \varepsilon\right)\right)\left(x-\left(z+\delta_{2} \varepsilon\right)\right)\left(x-\left(z+\delta_{3} \varepsilon\right)\right)
$$

obtained by perturbing the roots of the polynomial

$$
(x-z)^{3}=x^{3}-3 x^{2} z+3 x z^{2}-z^{3} .
$$

Using the basis $B=\left[1, x, x^{2}\right]$ we obtained the matrix of traces $R$, for which the $U$ matrix in the LU factorization obtained by GECP is

$$
\left(\begin{array}{ccc}
3 & 3 z+\varepsilon\left(\delta_{3}+\delta_{2}+\delta_{1}\right) & 3 z^{2}+\varepsilon\left(2 z \delta_{3}+2 \delta_{2} z+2 \delta_{1} z+\right)+\varepsilon^{2}\left(\delta_{3}^{2}+\delta_{2}^{2}+\delta_{1}^{2}\right) \\
0 & \frac{\varepsilon^{2}\left(-2 \delta_{1} \delta_{3}-2 \delta_{2} \delta_{3}-2 \delta_{1} \delta_{2}+2 \delta_{1}^{2}+2 \delta_{2}^{2}+2 \delta_{3}^{2}\right)}{3} & \frac{\varepsilon^{2} \Phi_{2,2}+\varepsilon^{3} \Phi_{2,3}}{\varepsilon^{3}} \\
0 & 0 & \frac{\varepsilon_{3,3}}{2\left(-\delta_{1} \delta_{3}-\delta_{2} \delta_{3}-\delta_{1} \delta_{2}+\delta_{1}^{2}+\delta_{2}^{2}+\delta_{3}^{2}\right)}
\end{array}\right)
$$

where $\Phi_{i, j}$ are polynomials in the $\delta$ 's and $z$ 's.

Using the bound from Proposition 5.8 for the numerical rank, we have that the approximate radical will be defined using the nullspace of the first row of $R$.

We obtain the following basis of the approximate radical,

$$
\left\{x^{2}-z^{2}-\frac{2 z \varepsilon\left(\delta_{3}+\delta_{2}+\delta_{1}\right)+\varepsilon^{2}\left(\delta_{3}^{2}+\delta_{2}^{2}+\delta_{1}^{2}\right)}{3}, x-z-\frac{\varepsilon\left(\delta_{3}+\delta_{2}+\delta_{1}\right)}{3}\right\}
$$


We choose the element of smallest degree to be the approximate square-free factor of $f$, which is here

$$
x-z-\frac{\varepsilon\left(\delta_{3}+\delta_{2}+\delta_{1}\right)}{3} .
$$

We can see that in this case the roots of this polynomial correspond precisely to the arithmetic mean of the three clustered roots.

(2) Consider the approximate polynomial

$$
\begin{gathered}
f(x)=(x+(-0.98816+0.01847 I))(x+(-0.98816-0.01847 I)) \\
(x-1.02390)(x-1.98603)(x-2.01375)
\end{gathered}
$$

which is a perturbation of the polynomial

$$
x^{5}-7 x^{4}+19 x^{3}-25 x^{2}+16 x-4=(x-1)^{3}(x-2)^{2} .
$$

The matrix of traces corresponding to $f$ is

$$
R=\left[\begin{array}{rrrrr}
5 & 7.00001 & 11.00013 & 19.00089 & 35.00425 \\
7.00001 & 11.00013 & 19.00089 & 35.00425 & 67.01631 \\
11.00013 & 19.00089 & 35.00425 & 67.01631 & 131.05456 \\
19.00089 & 35.00425 & 67.01631 & 131.05456 & 259.16598 \\
35.00425 & 67.01631 & 131.05456 & 259.16598 & 515.47172
\end{array}\right] .
$$

The $U_{k}$ matrix obtained after 2 steps of GECP on $R$ is

$$
U_{2}=\left[\begin{array}{rrrrr}
515.47172 & 35.00425 & 131.05456 & 259.16598 & 67.01631 \\
0 & 2.62296 & 2.10058 & 1.40165 & 2.44912 \\
0 & 0 & 0.0024342 & 0.0029279 & 0.0011698 \\
0 & 0 & 0.0029279 & 0.0035326 & 0.0014044 \\
0 & 0 & 0.0011698 & 0.0014044 & 0.00056307
\end{array}\right] .
$$

By taking the nullspace of the first two rows of the matrix $U_{2}$, we obtain the following basis of the approximate radical,

$$
\begin{aligned}
& \left\{x^{4}-15.01431 x+14.01921, x^{3}-7.00397 x+6.00539,\right. \\
& \left.x^{2}-3.00074 x+2.00102\right\} .
\end{aligned}
$$

The approximate square-free factor of $f$ is then

$$
x^{2}-3.00074 x+2.00102=(x-1.00028)(x-2.00047) .
$$

We can see that the roots of the output are close to the means of the clusters, and the differences are 0.00058 and 0.000200 respectively, which are of the order of the square of the cluster size (bounded here by 0.03 ).

We refer to the papers of [44, 21, 25, 51] for other methods that study approximate square-free factorization using approximate gcd computation.

\section{LU decomposition of the matrix of traces}

Since the polynomial system with clusters, obtained by perturbing the system with multiplicities, has only simple roots, the matrix of traces has full rank. However, we can try to find its numerical rank. We will argue below that we can define the numerical rank in such a way that it will be equal to the rank of the matrix of traces of the corresponding system with multiplicities. 
In this paper we primarily study the Gaussian elimination with complete pivoting (GECP) 20] in order to estimate the numerical rank and find the numerical nullspace of the matrix of traces. However we we will also infer that the singular value decomposition (SVD) in our case works similarly to the GECP.

We would like to note that rounding errors can sometimes result in a matrix which is close to a singular one, but where all the pivots are large (see Kahan's Example 5 in 23). This example shows that GECP can be a poor choice for numerical rank computations in the presence of rounding errors. On the other hand, algorithms for the accurate computations of the SVD of certain structured matrices, including Vandermonde matrices, use improved versions of GECP as subroutines [15, 16. In our case we prove that the structure of the matrix of traces guarantees that we will obtain small pivots which are proportional to the square of the size of the clusters and can therefore use GECP for rank determination.

We will also show how our results for the GECP of the matrix of traces $R$ relate to the singular values of $R$. In particular we will obtain asymptotic bounds for the singular values of the matrix $R$. Such bounds are similar to the ones for the entries of the $U_{k}$ matrix obtained after $k$ steps of GECP on $R$, more precisely, we also obtain in this case that the "almost zero" singular values are proportional to the square of the size of the clusters.

First we study the properties of the Gaussian elimination in the approximate setting. We use the following notation for different versions of the Gaussian elimination algorithm:

Definition 5.1. The version of Gaussian elimination in which at the $i$-th step we always select the entry at position $(i, i)$ for pivoting will be referred to as regular. We call an $m \times n$ matrix $M$ regular if for $k:=\operatorname{rank}(M)$ the first $k$ steps of the regular Gaussian elimination on $M$ do not encounter zero pivots.

Note that GECP on the matrix $M$ computes two permutation matrices $P$ and $Q$ of sizes $m \times m$ and $n \times n$, respectively, such that for the matrix $P M Q$ the regular Gaussian elimination works as GECP.

In the rest of this section we give results which compare the GECP applied to the matrices of traces of the perturbed system and to the system with multiple roots. Let $R_{0}$ be the matrix of traces of the system with multiple roots and let $R$ denote the matrix of traces of some perturbation of it. Assume that $\operatorname{rank}\left(R_{0}\right)=k$. Our next result guarantees that for sufficiently small clusters, the first $k$ steps of the GECP applied to $R$ computes permutation matrices $P$ and $Q$ which make the matrix $P R_{0} Q$ regular.

Proposition 5.2. Let $M$ be an $n \times n$ matrix with entries polynomials in $\mathbf{x}=$ $\left[x_{1}, \ldots, x_{N}\right]$ over $\mathbb{C}$. Fix $\mathbf{z}=\left[z_{1}, \ldots, z_{N}\right] \in \mathbb{C}^{N}$, denote $M_{0}:=\left.M\right|_{\mathbf{x}=\mathbf{z}}$, and assume that $\operatorname{rank}\left(M_{0}\right)=k$. Then there exists an open neighborhood $\mathcal{V}$ of $\mathbf{z}$ in $\mathbb{C}^{N}$ such that for all points $\tilde{\mathbf{z}}=\left[\tilde{z}_{1}, \ldots, \tilde{z}_{N}\right] \in \mathcal{V}$ if $P$ and $Q$ are the permutation matrices corresponding to the first $k$ steps of the GECP on the matrix $\tilde{M}:=\left.M\right|_{\mathbf{x}=\tilde{\mathbf{z}}}$, then the matrix $P M_{0} Q$ is regular. 
Proof. We call a pair $(P, Q)$ of $n$ by $n$ permutation matrices good if $P M_{0} Q$ is regular, otherwise the pair is called bad. For each bad pair we define an open neighborhood $\mathcal{V}_{P, Q}$ of $\mathbf{z} \in \mathbb{C}^{N}$ as follows: For some $i \leq k$ assume that the regular Gaussian elimination on $P M_{0} Q$ encounters a zero pivot for the first time in the $i$-th step, causing $(P, Q)$ to be a bad pair. Denote by $U_{0}$ the partially reduced form of $P M_{0} Q$ after the $i-1$-th step of the regular Gaussian elimination. Denote by $S$ the set of indices $(s, t)$ such that $s, t \geq i$ and the $(s, t)$ entry of $U_{0}$ is non-zero, and by $T$ the set of indices $(s, t)$ such that $s, t \geq i$ and the $(s, t)$ entry of $U_{0}$ is zero. Since the rank of $P M_{0} Q$ is $k, S$ is non empty.

Let $U$ be the partially reduced matrix obtained from $P M Q$ via the first $i-1$ steps of regular Gaussian elimination. Note that the entries of $U$ are rational functions of the entries of $M$ and the denominators of these are non zero at $\mathbf{z}$, hence are continuous functions of the points $\left[\tilde{z}_{1}, \ldots, \tilde{z}_{N}\right]$ in a sufficiently small neighborhood of $\mathbf{z}$. In particular, in an open neighborhood $\mathcal{U}$ of $\mathbf{z}$ the first $i-1$ steps of regular elimination can be carried out.

Let the open neighborhood $\mathcal{V}_{P, Q} \subset \mathcal{U} \subset \mathbb{C}^{N}$ of $\mathbf{z}$ be selected such that for all $\left[\tilde{z}_{1}, \ldots, \tilde{z}_{N}\right] \in \mathcal{V}_{P, Q}$ the entries in $T$ of $\tilde{U}:=\left.U\right|_{\mathbf{x}=\tilde{\mathbf{z}}}$ are all strictly smaller in absolute value than any of the entries in $S$ of $\tilde{U}$. By continuity, such open neighborhood of $\mathbf{z}$ exists, since the required inequalities hold for $U_{0}$.

Finally define $\mathcal{V}:=\bigcap_{(P, Q)} \mathcal{V}_{P, Q}$ is bad This is also an open neighborhood of $\mathbf{z}$ since the set of permutations is finite. We claim that for any fixed $\left[\tilde{z}_{1}, \ldots, \tilde{z}_{N}\right] \in \mathcal{V}$, if $(P, Q)$ is the pair of permutation matrices corresponding to the first $k$ steps of the Gaussian elimination with complete pivoting on the matrix $\tilde{M}$ then $(P, Q)$ is a good pair. This is true since $P \tilde{M} Q$ has the property that for each $i \leq k$ after $i-1$ steps of the Gauss elimination the $(i, i)$-th entry of the corresponding matrix is maximal in absolute value among the entries indexed by $(s, t) \neq(i, i)$ such that $s, t \geq i$. But then the $(i, i)$-th entry in the matrix $U_{0}$ defined above cannot be 0 because of the definition of $\mathcal{V}$. This proves the claim.

In the rest of the paper we will assume that the size of the clusters is a parameter $\varepsilon$. More precisely, in the following definition we formally explain the mathematical setting where our results will hold:

Definition 5.3. Let $\mathbf{z}_{i}=\left[z_{i, 1}, \ldots, z_{i, m}\right] \in \mathbb{C}^{m}$ for $i=1, \ldots, k$, and consider $k$ clusters $C_{1}, \ldots, C_{k}$ of size $\left|C_{i}\right|=n_{i}$ such that $\sum_{i=1}^{k} n_{i}=n$, each of radius proportional to the parameter $\varepsilon$ in the $\infty$-norm around $\mathbf{z}_{1}, \ldots, \mathbf{z}_{k}$ :

$$
\begin{aligned}
C_{i}= & \left\{\left[z_{i, 1}+\delta_{i, 1,1} \varepsilon, \ldots, z_{i, m}+\delta_{i, 1, m} \varepsilon\right], \ldots,\right. \\
& \left.\ldots\left[z_{i, 1}+\delta_{i, n_{i}, 1} \varepsilon, \ldots, z_{i, m}+\delta_{i, n_{i}, m} \varepsilon\right]\right\} \\
= & \left\{\mathbf{z}_{i}+\vec{\delta}_{i, 1} \varepsilon, \ldots, \mathbf{z}_{i}+\vec{\delta}_{i, n_{i}} \varepsilon\right\},
\end{aligned}
$$

where $\left|\delta_{i, j, r}\right|<1$ for all $i=1, \ldots, k, j=1, \ldots, n_{i}, r=1, \ldots, m$. Let $U_{k}$ be the partially row reduced form obtained by applying $k$ steps of the GECP to the matrix of traces $R$ corresponding to $C_{1} \cup \cdots \cup C_{k}$. Then $R$ and $U_{k}$ have entries from the field $\mathbb{C}(\varepsilon)$. 
Assumption 5.4. Based on Proposition 5.2, we will assume that if the GECP applied to $R$ produces the permutation matrices $P$ and $Q$ then the matrix $P R_{0} Q$ is regular, where $R_{0}=\left.R\right|_{\varepsilon=0}$. To simplify the notation for the rest of the paper we will assume that $Q=i d$, i.e. the rows and columns of $P R Q=P R$ correspond to the bases

$$
\sigma B=\left[b_{\sigma(1)}, \ldots, b_{\sigma(n)}\right] \text { and } B=\left[b_{1}, \ldots, b_{n}\right],
$$

respectively, where $\sigma$ is the permutation corresponding to the matrix $P$. This assumption does not constrain the generality since we may rename $B$ in the definition of $R$.

With the assumption that $P R_{0}$ has rank $k$ and is regular, we can assume that all the denominators appearing in the entries of $U_{k}$ are minors of $R$ which are non-zero at $\varepsilon=0$. Therefore we can take their Taylor expansion around $\varepsilon=0$ and consider them as elements of the formal power series ring $\mathbb{C}[[\varepsilon]]$. In this ring we shall work with residue classes modulo $\varepsilon^{2}$, i.e., in some considerations we factor out the ideal $\left\langle\varepsilon^{2}\right\rangle$ of $\mathbb{C}[[\varepsilon]]$.

The results in the rest of the paper are all valid modulo $\varepsilon^{2}$ in the formal power series setting described above. In practice what this means is that the method we propose works up to a precision which is the double of the original size of the clusters.

Remark 5.5. In Definition 5.3 we assume that the clusters are linear perturbations of a set of multiple roots. Note that not all multiplicity structures can be obtained as a limit of such clusters with linear perturbation of fixed directions $\vec{\delta}_{i, j}$ as $\varepsilon$ approaches 0. However, as we have seen in Proposition 3.4 the matrix of traces at $\varepsilon=0$ is independent of the directions $\vec{\delta}_{i, j}$, and in fact does not depend on the multiplicity structure of the roots. Since all the subsequent results in the paper only depend on the matrix of traces and are only valid modulo $\varepsilon^{2}$, we do not limit the generality by considering only linear perturbations. This is not true however for the multiplication matrices, which depend on the multiplicity structure of the roots at $\varepsilon=0$, as seen in Example 3.2

In order to describe the structure of the matrices in the $L U$ decomposition of the matrix of traces obtained by GECP in terms of the elements in the clusters, we need the following definition:

Definition 5.6. Let $B=\left[b_{1}, \ldots, b_{n}\right] \in \mathbb{C}\left[x_{1}, \ldots, x_{m}\right]^{n}$, and let $\mathbf{z}_{1}, \ldots, \mathbf{z}_{r} \in \mathbb{C}^{m}$ be not necessary distinct points. We call the $n \times r$ matrix

$$
V:=\left[b_{i}\left(\mathbf{z}_{j}\right)\right]_{i, j=1}^{n, r}
$$

the Vandermonde matrix of $\mathbf{z}_{1}, \ldots, \mathbf{z}_{r}$ w.r.t. $B$. Note that if $r=n$ then the matrix of traces in Definition 0 and the Vandermonde matrix are closely related:

$$
R=V V^{T} \text {. }
$$


The following proposition gives asymptotic bounds for the entries of the matrix obtained from a partial Gauss elimination with complete pivoting on the matrix of traces $R$ for the case where the $n$ roots of the system correspond to $k$ clusters, each of them with $n_{i}$ roots $(i=1, \ldots, k)$ and radius proportional to $\varepsilon$ in the max-norm.

Proposition 5.7. Let $B=\left[b_{1}, \ldots, b_{n}\right] \in \mathbb{C}\left[x_{1}, \ldots, x_{m}\right]^{n}$. Let $\left\{\mathbf{z}_{1}, \ldots, \mathbf{z}_{k}\right\} \in \mathbb{C}^{m}$ and the clusters $C_{1}, \ldots, C_{k}$ around $\left\{\mathbf{z}_{1}, \ldots, \mathbf{z}_{k}\right\}$ be as in Definition 5.3 .

Let $R$ be the matrix of traces associated to $C_{1} \cup \cdots \cup C_{k}$ and $B$ (see Definition 2.4 and Proposition 3.4). Let $P$ and $R_{0}:=\left.R\right|_{\varepsilon=0}$ be as in Assumption 5.4 and assume that $P R_{0}$ has rank $k$ and is regular. Then, after $k$ steps of the regular Gaussian elimination on $P R$ we get a partially row reduced matrix $U_{k}$, such that its last $n-k$ rows satisfy

$$
\left[U_{k}\right]_{i, j}=\left\{\begin{array}{ll}
0, & \text { if } j \leq k \\
c_{i, j} \varepsilon^{2}+\text { h.o.t. }(\varepsilon) \quad \in \mathbb{C}[[\varepsilon]] & \text { if } j>k
\end{array} \text { for } i=k+1, \ldots, n .\right.
$$

The values of $c_{i, j} \in \mathbb{C}$ depends on $n,\left\{\mathbf{z}_{1}, \ldots, \mathbf{z}_{k}\right\},\left\{\vec{\delta}_{s, t}\right\}$ and $B$ (we will give a bound for $c_{i, j}$ in Proposition [5.8). Here h.o.t.( $(\varepsilon)$ denotes the higher order terms in $\varepsilon$. Moreover, the formal power series in (6) are convergent in a sufficiently small neighborhood of $\varepsilon=0$.

Proof. To simplify the notation, denote $\tilde{R}=P R$. The proof is based on the fact that after $k$ steps of the regular Gaussian elimination on $\tilde{R}$, the partially reduced $U_{k}$ matrix has elements $(i, j)$, for $i, j=k+1, \ldots, n$, of the form

$$
\frac{\operatorname{det}\left(\tilde{R}_{i, j}^{(k+1)}\right)}{\operatorname{det}\left(\tilde{R}^{(k)}\right)}
$$

where $\tilde{R}^{(k)}$ is the $k \times k$ principal submatrix of $\tilde{R}$ and $\tilde{R}_{i, j}^{(k+1)}$ is the $(k+1) \times(k+1)$ submatrix of $\tilde{R}$ corresponding to rows $\{1, \ldots, k, i\}$ and columns $\{1, \ldots, k, j\}$. This follows at once from the facts that both the numerator and the denominator of (8) stay the same during the row operations performed, and the reduced form of $R_{i, j}^{(k+1)}$ is upper triangular.

Let $V$ be the $n \times n$ Vandermonde matrix of $C_{1} \cup \cdots \cup C_{k}$ with respect to $B$ and recall that $R=V V^{T}$, thus $\tilde{R}=(P V)\left(V^{T}\right)$. Let $\sigma$ be the permutation corresponding to $P$ and let $\sigma B$ be as in (5). Observe that

$$
\tilde{R}_{i, j}^{(k+1)}=V_{\sigma B_{i}} V_{B_{j}}^{T}
$$

where $V_{\sigma B_{i}}$ and $V_{B_{j}}$ are the $(k+1) \times n$ Vandermonde matrices corresponding to $C_{1} \cup \cdots \cup C_{k}$ and respectively to $\sigma B_{i}:=\left[b_{\sigma(1)}, \ldots, b_{\sigma(k)}, b_{\sigma(i)}\right]$, and $B_{j}:=$ $\left[b_{1}, \ldots, b_{k}, b_{j}\right]$. Therefore, by the Cauchy-Binet formula we have

$$
\operatorname{det}\left(\tilde{R}_{i, j}^{(k+1)}\right)=\sum_{|I|=k+1} \operatorname{det}\left(V_{\sigma B_{i}, I}\right) \operatorname{det}\left(V_{B_{j}, I}\right),
$$

where $V_{\sigma B_{i}, I}$ denotes the $(k+1) \times(k+1)$ submatrix of $V_{\sigma B_{i}}$ with columns corresponding to the points in $I \subset C_{1} \cup \cdots \cup C_{k}$, and the summation is taken for all $I \subset$ 
$C_{1} \cup \cdots \cup C_{k}$ such that $|I|=k+1$. Note that all the determinants in (8) are polynomials in $\varepsilon$. Since $\operatorname{rank}\left(\left.V\right|_{\varepsilon=0}\right)=k$, we have $\left.\operatorname{det}\left(V_{\sigma B_{i}, I}\right)\right|_{\varepsilon=0}=\left.\operatorname{det}\left(V_{B_{j}, I}\right)\right|_{\varepsilon=0}=0$, thus they are divisible by $\varepsilon$ for all $i=k+1, \ldots n$ and $I \subset C_{1} \cup \cdots \cup C_{k}$ with $|I|=k+1$. Therefore we get that $\operatorname{det}\left(\tilde{R}_{i, j}^{(k+1)}\right)$ is divisible by $\varepsilon^{2}$.

Finally we note that the assumption that $P R_{0}$ has rank $k$ and is regular implies that

$$
\operatorname{det}\left(\left.\tilde{R}^{(k)}\right|_{\varepsilon=0}\right) \neq 0,
$$

which proves that the Taylor expansion of the ratio in (11) around $\varepsilon=0$ has zero constant and linear terms, as was claimed. The formal power series in (6) are convergent in a sufficiently small neighborhood of $\varepsilon=0$, since they are the Taylor series of rational functions with non-zero denominators at $\varepsilon=0$.

From the previous results it follows that if we have $k$ clusters of size $n_{i}$, with $i=1, \ldots, k, \sum_{i=1}^{k} n_{i}=n$, then after $k$ steps of GECP on the matrix of traces $R$, we get the matrix

$$
U_{k}=\left[\begin{array}{cccccc}
{\left[U_{k}\right]_{1,1}} & & \cdots & \cdots & \cdots & {\left[U_{k}\right]_{1, n}} \\
0 & \ddots & \cdots & \cdots & \cdots & \vdots \\
& & {\left[U_{k}\right]_{k, k}} & \cdots & \cdots & {\left[U_{k}\right]_{k, n}} \\
\vdots & & 0 & c_{k+1, k+1} \varepsilon^{2} & \cdots & c_{k+1, n} \varepsilon^{2} \\
& & \vdots & \vdots & \ddots & \vdots \\
0 & & 0 & c_{n, k+1} \varepsilon^{2} & \cdots & c_{n, n} \varepsilon^{2}
\end{array}\right]+\text { h.o.t. }(\varepsilon)
$$

where the constant term in $\varepsilon$ of $\left[U_{k}\right]_{i, i}$ is non-zero for $i \leq k$.

The next proposition gives a bound for the coefficient $c_{i, j}$ of $\varepsilon^{2}$ in (9). It also gives an idea of the magnitude of the threshold one can use to decide on the numerical rank which would additionally indicate how small the size of the clusters need to be for our method to work.

Proposition 5.8. Let $B=\left[b_{1}, \ldots, b_{n}\right] \in \mathbb{C}\left[x_{1}, \ldots, x_{m}\right]^{n}$. Let $\left\{\mathbf{z}_{1}, \ldots, \mathbf{z}_{k}\right\} \in \mathbb{C}^{m}$. Let the clusters $C_{1}, \ldots, C_{k}$ around $\left\{\mathbf{z}_{1}, \ldots, \mathbf{z}_{k}\right\}$ be as in (4) with $\left|\delta_{i, j, r}\right| \leq 1$ for all $i=1, \ldots, k, j=1, \ldots, n_{i}, r=1, \ldots, m$. Let $R$ be the matrix of traces associated to $C_{1} \cup \cdots \cup C_{k}$ and $B$.

Let $\mathbf{b}^{\prime}$ be such that

$$
\mathbf{b}^{\prime} \geq \max _{\{l, i, r\}}\left\{\left|\frac{\partial b_{l}}{\partial x_{r}}\left(\mathbf{z}_{i}\right)\right|\right\} .
$$

Assume that the GECP applied to $R$ also implies complete pivoting on $\left.R\right|_{\varepsilon=0}$. Then the bound for the coefficients $c_{i, j}$ of $\varepsilon^{2}$ in the $U_{k}$ matrix, obtained after $k$ steps of the GECP applied to the matrix of traces $R$, is given by

$$
\left|c_{i, j}\right| \leq \alpha \cdot\left(\mathbf{b}^{\prime}\right)^{2},
$$

where $\alpha=4(n-k)(k+1)^{2} m^{2}$. 
Proof. We denote $\tilde{R}:=P R$, where $P$ is a permutation matrix such that the first $k$ steps of GECP applied to both $P R$ and $\left.P R\right|_{\varepsilon=0}$ is well defined and the same as regular Gaussian elimination. Note that we need the assumption that GECP applied to $R$ also implies complete pivoting on $\left.R\right|_{\varepsilon=0}$ since Proposition 5.2 only implies that $\left.P R\right|_{\varepsilon=0}$ is regular, but below we will also need the pivots in $\left.P R\right|_{\varepsilon=0}$ to have maximal absolute values. One can achieve this by making the right selection among equal possible pivots while performing GECP on $R$. We will use this assumption at the end of the proof.

Denote the bases corresponding to the rows and columns of $\tilde{R}$ by $\sigma B=$ $\left[b_{\sigma(1)}, \ldots, b_{\sigma(n)}\right]$ and $B=\left[b_{1}, \ldots, b_{n}\right]$ as in (5). the form

The partially reduced $U_{k}$ matrix has elements $(i, j)$, for $i, j=k+1, \ldots, n$, of

$$
\frac{\operatorname{det}\left(\tilde{R}_{B^{\prime}, B^{\prime \prime}}^{(k+1)}\right)}{\operatorname{det}\left(\tilde{R}^{(k)}\right)}
$$

where $\tilde{R}^{(k)}$ is the $k$-th principal submatrix of $\tilde{R}$ and $\tilde{R}_{B^{\prime}, B^{\prime \prime}}^{(k+1)}$ is the $(k+1) \times(k+1)$ submatrix of $\tilde{R}$ corresponding to rows $B^{\prime}:=\left[b_{\sigma(1)}, \ldots, b_{\sigma(k)}, b_{\sigma(i)}\right]$ and columns $B^{\prime \prime}:=\left[b_{1}, \ldots, b_{k}, b_{j}\right]$. In order to get an upper bound for $\left|c_{i, j}\right|$, we will get an upper bound for the coefficient of $\varepsilon^{2}$ in $\operatorname{det}\left(\tilde{R}_{B^{\prime}, B^{\prime \prime}}^{(k+1)}\right)$ and divide it by the constant term of $\left|\operatorname{det}\left(\tilde{R}^{(k)}\right)\right|$.

Fix $i, j \in\{1, \ldots, n\}$. We will use the Cauchy-Binet formula

$$
\operatorname{det}\left(\tilde{R}_{B^{\prime}, B^{\prime \prime}}^{(k+1)}\right)=\sum_{|I|=k+1} \operatorname{det}\left(V_{B^{\prime}, I}\right) \operatorname{det}\left(V_{B^{\prime \prime}, I}\right),
$$

where the summation is for $I \subset C_{1} \cup \cdots \cup C_{k}$ of cardinality $k+1$, and $V_{B^{\prime}, I}$ and $V_{B^{\prime \prime}, I}$ are the Vandermonde matrices corresponding to $I$ w.r.t. $B^{\prime}$ and $B^{\prime \prime}$, respectively. Since the derivative of the determinant of a matrix is the sum of determinants obtained by replacing one by one the columns by the derivative of that column, after expanding the determinants in the sum by their columns containing the derivatives, we get

$$
\left.\frac{\partial \operatorname{det}\left(V_{B^{\prime}, I}\right)}{\partial \varepsilon}\right|_{\varepsilon=0}=\sum_{b^{\prime} \in B^{\prime}} \sum_{\mathbf{z} \in I} \pm\left.\left(\left.\sum_{t=1}^{m} \delta_{\mathbf{z}, t} \frac{\partial b^{\prime}}{\partial x_{t}}(\mathbf{z})\right|_{\varepsilon=0}\right) \operatorname{det}\left(V_{B^{\prime}-\left\{b^{\prime}\right\}, I-\{\mathbf{z}\}}\right)\right|_{\varepsilon=0},
$$

where $\delta_{\mathbf{z}, t}$ is the coefficient of $\varepsilon$ in the $t$-th coordinate of $\mathbf{z} \in I$. We can obtain a similar expression for $\left.\frac{\partial \operatorname{det}\left(V_{B^{\prime \prime}, I}\right)}{\partial \varepsilon}\right|_{\varepsilon=0}$.

Note that $\left.\operatorname{det}\left(V_{B^{\prime}-\{b\}, I-\{\mathbf{z}\}}\right)\right|_{\varepsilon=0}$ is non-zero only if $\left.I\right|_{\varepsilon=0}=\left\{\mathbf{z}_{1}, \ldots, \mathbf{z}_{k}\right\} \cup$ $\left\{\mathbf{z}_{i}\right\}$ for some $i=1, \ldots, k$ and $\left.\mathbf{z}\right|_{\varepsilon=0}=\mathbf{z}_{i}$. In that case $I-\left.\{\mathbf{z}\}\right|_{\varepsilon=0}=\left\{\mathbf{z}_{1}, \ldots, \mathbf{z}_{k}\right\}$, which we denote by

for simplicity.

$$
\mathfrak{Z}:=\left\{\mathbf{z}_{1}, \ldots, \mathbf{z}_{k}\right\}
$$

Thus we have that if $\left.I\right|_{\varepsilon=0}=\left\{\mathbf{z}_{1}, \ldots, \mathbf{z}_{k}\right\} \cup\left\{\mathbf{z}_{i}\right\}$ then 


$$
\left|\frac{\partial \operatorname{det}\left(V_{B^{\prime}, I}\right)}{\partial \varepsilon}\right|_{\varepsilon=0} \leq \sum_{b^{\prime} \in B^{\prime}} \sum_{\substack{\mathbf{z} \in I \\ \mathbf{z} \mid \varepsilon=0=\mathbf{z}_{i}}}\left|\operatorname{det}\left(V_{B^{\prime}-\left\{b^{\prime}\right\}, \mathcal{Z}}\right)\right| \sum_{t=1}^{m}\left|\frac{\partial b^{\prime}}{\partial x_{t}}(\mathbf{z})\right|_{\varepsilon=0}
$$

using that $\left|\delta_{\mathbf{z}, t}\right| \leq 1$. Therefore, we get that the coefficient of $\varepsilon^{2}$ in $\operatorname{det}\left(\tilde{R}_{B^{\prime}, B^{\prime \prime}}^{(k+1)}\right)$ is bounded by

$$
\begin{aligned}
4 \sum_{\substack{b^{\prime} \in B^{\prime} \\
b^{\prime \prime} \in B^{\prime \prime}}}\left|\operatorname{det}\left(V_{B^{\prime}-\left\{b^{\prime}\right\}, \mathfrak{Z}}\right) \operatorname{det}\left(V_{B^{\prime \prime}-\left\{b^{\prime \prime}\right\}, \boldsymbol{Z}}\right)\right| \\
\left(\sum_{\substack{|I|=k+\left.1 \\
I\right|_{\varepsilon}=0=\left\{\mathbf{z}_{1}, \ldots, \mathbf{z}_{k}\right\} \cup\left\{\mathbf{z}_{i}\right\}}}\left(\sum_{t=1}^{m}\left|\frac{\partial b^{\prime}}{\partial x_{t}}\left(\mathbf{z}_{i}\right)\right|\right)\left(\sum_{t=1}^{m}\left|\frac{\partial b^{\prime \prime}}{\partial x_{t}}\left(\mathbf{z}_{i}\right)\right|\right)\right) .
\end{aligned}
$$

using the fact that there are two possible ways to pick $\mathbf{z} \in I$ with $\left.\mathbf{z}\right|_{\varepsilon=0}=\mathbf{z}_{i}$ from $\left.I\right|_{\varepsilon=0}=\left\{\mathbf{z}_{1}, \ldots, \mathbf{z}_{k}\right\} \cup\left\{\mathbf{z}_{i}\right\}$.

Using the upper bound $\mathbf{b}^{\prime}$ and counting the number of times we can choose $I \subset C_{1} \cup \cdots \cup C_{k}$ such that $|I|=k+1$ and $\left.I\right|_{\varepsilon=0}=\left\{\mathbf{z}_{1}, \ldots, \mathbf{z}_{k}\right\} \cup\left\{\mathbf{z}_{i}\right\}$, we get

$$
4 m^{2} \mathbf{b}^{\prime 2}(n-k)\left(\prod_{j=1}^{k} n_{j}\right)\left(\sum_{\substack{b^{\prime} \in B^{\prime} \\ b^{\prime \prime} \in B^{\prime \prime}}}\left|\operatorname{det}\left(V_{B^{\prime}-\left\{b^{\prime}\right\}, \mathfrak{Z}}\right) \operatorname{det}\left(V_{B^{\prime \prime}-\left\{b^{\prime \prime}\right\}, \boldsymbol{Z}}\right)\right|\right) .
$$

On the other hand, we have that if $\tilde{R}_{B^{\prime}-\left\{b^{\prime}\right\}, B^{\prime \prime}-\left\{b^{\prime \prime}\right\}}$ is the matrix of traces with rows corresponding to the $B^{\prime}-\left\{b^{\prime}\right\}$ and columns corresponding to $B^{\prime \prime}-\left\{b^{\prime \prime}\right\}$ then

$$
\left.\operatorname{det}\left(\tilde{R}_{B^{\prime}-\left\{b^{\prime}\right\}, B^{\prime \prime}-\left\{b^{\prime \prime}\right\}}\right)\right|_{\varepsilon=0}=\left(\prod_{i=1}^{k} n_{i}\right) \operatorname{det}\left(V_{B^{\prime}-\left\{b^{\prime}\right\}, \mathfrak{Z}}\right) \operatorname{det}\left(V_{B^{\prime \prime}-\left\{b^{\prime \prime}\right\}, \boldsymbol{Z}}\right) .
$$

Therefore, the bound for the coefficient of $\varepsilon^{2}$ in $\operatorname{det}\left(\tilde{R}_{B^{\prime}, B^{\prime \prime}}^{(k+1)}\right)$ is

$$
4 m^{2} \mathbf{b}^{\prime 2}(n-k) \sum_{\substack{b^{\prime} \in B^{\prime} \\ b^{\prime \prime} \in B^{\prime \prime}}}\left|\operatorname{det}\left(\tilde{R}_{B^{\prime}-\left\{b^{\prime}\right\}, B^{\prime \prime}-\left\{b^{\prime \prime}\right\}}\right)\right|_{\varepsilon=0} .
$$

Next we use the assumption above on $\left.\tilde{R}\right|_{\varepsilon=0}=\left.P R\right|_{\varepsilon=0}$ to have maximal pivots in the first $k$ diagonal entries to get

$$
\left|\operatorname{det}\left(\tilde{R}^{(k)}\right)\right|_{\varepsilon=0} \geq\left|\operatorname{det}\left(\tilde{R}_{B^{\prime}-\left\{b^{\prime}\right\}, B^{\prime \prime}-\left\{b^{\prime \prime}\right\}}\right)\right|_{\varepsilon=0}
$$

which is true since the left and right hand side of (13) divided by $\left|\operatorname{det}\left(\tilde{R}^{(k-1)}\right)\right|_{\varepsilon=0}$ give the absolute values of the entries of the partially row reduced matrix after $k-1$ steps of GECP. Therefore we can replace $\left|\operatorname{det}\left(\tilde{R}_{B^{\prime}-\left\{b^{\prime}\right\}, B^{\prime \prime}-\left\{b^{\prime \prime}\right\}}\right)\right|_{\varepsilon=0}$ by 
$\left|\operatorname{det}\left(\tilde{R}^{(k)}\right)\right|_{\varepsilon=0}$ in (12) and divide the expression (12) by $\left|\operatorname{det}\left(\tilde{R}^{(k)}\right)\right|_{\varepsilon=0}$, thus we get the following bound for the coefficient $c_{i, j}$ of $\varepsilon^{2}$ in the $U_{k}$ matrix:

$$
4 m^{2} \mathbf{b}^{\prime 2}(n-k)(k+1)^{2} .
$$

Remark 5.9. The above proposition gives estimates in terms of $\left\{\mathbf{z}_{1}, \ldots, \mathbf{z}_{k}\right\}$, which we do not assume to know a priori. The following heuristic methods can be used to check whether the estimated numerical rank is correct, given a required precision $\varepsilon$. Assuming that we know the magnitude of the coordinates of the roots, we may compute the matrix of traces corresponding to $n$ randomly chosen distinct roots which have the same order of magnitude as the original roots. Then comparing the diagonal entries of the $U$-matrices obtained by applying the GECP for the matrices of traces, we can set the numerical rank to be the first entry where the discrepancy is of order $\varepsilon^{2}$. Another heuristics is to increase $k$ one by one, compute the approximate radical ideal (see Definition 17.1) corresponding to the case when the numerical rank of $R$ is $k$. Compute the roots of the approximate radical ideal, and substitute them back into the original system. If the error is of order of magnitude $\varepsilon$, accept $k$ and the computed approximate radical as the output.

Example 5.10. Continuing Example [3.3, we apply the GECP to the matrix $R$ defined in (3). After two steps of GECP we obtain the following matrix:

$$
U_{2}=\left[\begin{array}{ccccc}
11.07093 & -5.04240 & 7.03226 & -1.01900 & -1.04951 \\
0 & 8.71265 & 2.18381 & 6.53716 & 6.55387 \\
0 & 0 & 0.454213 \times 10^{-4} & 0.7407 \times 10^{-5} & 0.178036 \times 10^{-3} \\
0 & 0 & 0.7397 \times 10^{-5} & 0.728 \times 10^{-6} & 0.41955 \times 10^{-4} \\
0 & 0 & 0.188071 \times 10^{-3} & 0.52002 \times 10^{-4} & 0.657084 \times 10^{-3}
\end{array}\right]
$$

with columns permuted so that they correspond to the basis $\left[x_{1} x_{2}, x_{2}, x_{1}, 1, x_{1}^{2}\right]$. Note that the largest entry in the $3 \times 3$ bottom right corner of $U_{2}$ is between $\varepsilon$ and $\varepsilon^{2}$ (here $\varepsilon \approx 0.01$ in this example). Thus we consider the numerical rank of $R$ to be 2. From the nullspace of the first two rows of $U_{2}$ we can obtain the following approximate multiplication matrices:

$$
\mathcal{M}_{x_{1}}^{\prime}=\left[\begin{array}{cc}
0 & 1 \\
1.00382 & -0.37849 \times 10^{-3}
\end{array}\right] \quad \mathcal{M}_{x_{2}}^{\prime}=\left[\begin{array}{cc}
1.49973 & -0.49972 \\
-0.5016325 & 1.50162
\end{array}\right]
$$

(see Section 7 below for more details on approximate multiplication matrices). The eigenvalues of $\mathcal{M}_{x_{1}}^{\prime}$ and $\mathcal{M}_{x_{2}}^{\prime}$ are respectively

$$
\{1.000018,-1.003803\} \text { and }\{0.9999943,2.001349\} \text {. }
$$

Note that these eigenvalues are close to the avarages of the coordinates of the roots in the two clusters.

\section{Singular Values of $R$}

Using the previous results we will now study the singular values of the matrix of traces $R$ of a system with clusters of roots. We denote $\tilde{R}:=P R$, where $P$ is a permutation matrix obtained by $k$ steps of GECP applied to $R$ and we assume 
that $\left.P R\right|_{\varepsilon=0}$ is regular, as in Assumption 5.4 Let $U_{k}$ be the matrix obtained after $k$ steps of GECP on the matrix $\tilde{R}$, as in (9). Let $\widehat{U}_{k}$ be the matrix obtained after replacing the last $n-k$ rows of $U_{k}$ by zeros. Let $L_{k}$ be such that $\tilde{R}=L_{k} U_{k}$ (in other words $L_{k}$ is the transformation matrix obtained after $k$ steps of GECP on $\tilde{R})$. Let $\widehat{R}=L_{k} \widehat{U}_{k}$. Using the submultiplicative property of matrix norms, we have that

$$
\|\tilde{R}-\widehat{R}\|_{F}=\left\|L_{k} U_{k}-L_{k} \widehat{U}_{k}\right\|_{F} \leq\left\|L_{k}\right\|_{F}\left\|U_{k}-\widehat{U}_{k}\right\|_{F}
$$

where $\|\cdot\|_{F}$ denotes the Frobenius matrix norm.

Let $\sigma_{1} \geq \cdots \geq \sigma_{n}$ be the singular values of $R$, which are also the singular values of $\tilde{R}$. Since by definition $\sigma_{i}$ is the 2 -norm distance from $\tilde{R}$ to the nearest rank $i$ matrix, and $\widehat{R}$ is an $n$ by $n$ matrix of rank $k$, we have that

$$
\sigma_{n} \leq \cdots \sigma_{k+1} \leq\|\tilde{R}-\widehat{R}\|_{2} .
$$

Given that the 2-norm of a matrix is smaller than or equal to its Frobenius norm, we have

$$
\sigma_{n} \leq \cdots \sigma_{k+1} \leq\left\|L_{k}\right\|_{F}\left\|U_{k}-\widehat{U}_{k}\right\|_{F}
$$

Since we are using GECP it is easy to see that

$$
\left[L_{k}\right]_{i, j} \leq 1 \text { for all } j=1, \ldots, k, i>j
$$

and the matrix $L_{k}$ obtained after $k$ steps of GECP is of the form

$$
L_{k}=\left[\begin{array}{ccccccc}
1 & 0 & \cdots & \cdots & \cdots & \cdots & 0 \\
{\left[L_{k}\right]_{2,1}} & \ddots & \ddots & \cdots & \cdots & \cdots & \vdots \\
\vdots & \ddots & 1 & 0 & \cdots & \cdots & \vdots \\
\vdots & \cdots & {\left[L_{k}\right]_{k+1, k}} & 1 & 0 & \cdots & \vdots \\
\vdots & \cdots & \vdots & 0 & \ddots & \ddots & \vdots \\
\vdots & \cdots & \vdots & \vdots & \ddots & \ddots & 0 \\
{\left[L_{k}\right]_{n, 1}} & \cdots & {\left[L_{k}\right]_{n, k}} & 0 & \cdots & 0 & 1
\end{array}\right]
$$

Therefore we have

$$
\left\|L_{k}\right\|_{F} \leq \sqrt{\frac{2 n+2 n k-k^{2}-k}{2}} .
$$

From Proposition 5.8 we have that for $i, j=k+1 \ldots n$, the elements of $U_{k}$ are of the form

$$
\left[U_{k}\right]_{i j}=\omega \varepsilon^{2}+\text { h.o.t. }(\varepsilon),
$$

where $\omega=4(n-k)(k+1)^{2} m^{2} \mathbf{b}^{\prime 2}$ and $\mathbf{b}^{\prime 2}$ is defined in (10).

We therefore have

$$
\left\|U_{k}-\widehat{U}_{k}\right\|_{F}=\sqrt{\sum_{i, j=k+1}^{n}\left(\left[U_{k}\right]_{i j}\right)^{2}} \leq(n-k) \omega \varepsilon^{2}+\text { h.o.t. }(\varepsilon) .
$$


We summarize the above argument in the next Proposition, showing that the $k+1$-th singular value of $R$ is asymptotically equal to $\varepsilon^{2}$.

Proposition 6.1. Let $R$ be the matrix of traces associated to $C_{1} \cup \cdots \cup C_{k}$ and $B$ where the clusters $C_{1}, \ldots, C_{k}$ around $\mathbf{z}_{1}, \ldots, \mathbf{z}_{k} \in \mathbb{C}^{m}$ are as in (4) and $B=$ $\left[b_{1}, \ldots, b_{n}\right] \in \mathbb{C}\left[x_{1}, \ldots, x_{m}\right]^{n}$. Let $\sigma_{1} \geq \cdots \geq \sigma_{n}$ be the singular values of $R$. Then

$$
\sigma_{k+1}=\Omega \varepsilon^{2}+\text { h.o.t. }(\varepsilon)
$$

where

$$
\Omega \leq 4(n-k)^{2}(k+1)^{2} m^{2} \sqrt{\frac{2 n+2 n k-k^{2}-k}{2}}\left(\mathbf{b}^{\prime}\right)^{2}
$$

and $\mathbf{b}^{\prime}$ is defined in (10).

Example 6.2. Continuing Example 3.3, we compute the singular values of the matrix $R$ defined in (3):

$$
\left[22.8837,14.2433,0.448334 \times 10^{-3}, 0.174904 \times 10^{-4}, 0.594796 \times 10^{-5}\right] .
$$

We have that the third singular value is between $\varepsilon$ and $\varepsilon^{2}$ (in this example $\varepsilon \approx 0.01$ ), thus we can set the numerical rank of the matrix $R$ to be 2. Note that the 2-norm distance of the matrix $R$ from $\left.R\right|_{\varepsilon=0}$ is not the same order of magnitude as the third singular value, it is 0.147 as was computed in Example 3.3. This is the reason why we used the partial $L U$-decomposition of $R$ and not $\left.R\right|_{\varepsilon=0}$ to obtain a bound for $\sigma_{k+1}$.

\section{Approximate Radical Ideal}

Using our previous results, we can now define the concept of an approximate radical ideal and describe its roots in terms of the elements of the clusters.

Definition 7.1. Let $B=\left[b_{1}, \ldots, b_{n}\right] \in \mathbb{C}\left[x_{1}, \ldots, x_{m}\right]^{n}$ and the clusters $C_{1}, \ldots, C_{k}$ be as in Definition 5.9. Let $R$ be the matrix of traces associated to $C_{1} \cup \cdots \cup C_{k}$ and $B$. Let the permutation matrix $P$ corresponding to the permutation $\sigma$ obtained after $k$ steps of GECP on $R$ as in Assumption 5.4, so that the rows and columns of $\tilde{R}:=P R$ correspond to $\sigma B$ and $B$, respectively, as in (5). We define the vectors $\mathbf{v}_{i, j} \in \mathbb{C}(\varepsilon)^{k}$ for $i=1, \ldots m$ and $j=1, \ldots k$, as the solutions of the following $m k$ linear systems:

$$
\tilde{R}^{(k)} \mathbf{v}_{i, j}=\mathbf{r}_{i, j} \quad i=1, \ldots, m \quad j=1, \ldots, k,
$$

where the left hand sides are always the $k \times k$ principal submatrix of $\tilde{R}$, while for any fixed $i$ and $j$ the right hand side of (14) is defined as

$$
\mathbf{r}_{i, j}:=\left[\begin{array}{c}
\operatorname{Tr}\left(x_{i} b_{j} b_{\sigma(1))}\right. \\
\vdots \\
\operatorname{Tr}\left(x_{i} b_{j} b_{\sigma(k)}\right)
\end{array}\right] \in \mathbb{C}^{k} .
$$

Note that one can compute the vectors $\mathbf{r}_{i, j}$ the same way as the columns of the matrix of traces. Then we define the following $m k$ polynomials: 


$$
f_{i, j}:=x_{i} b_{j}-\left(\sum_{s=1}^{k}\left[\mathbf{v}_{i, j}\right]_{s} b_{s}\right) \quad i=1, \ldots, m, \quad j=1, \ldots, k .
$$

We will call the approximate radical ideal of the clusters $C_{1} \cup \cdots \cup C_{k}$ the ideal generated by

$$
\widetilde{\sqrt{I}}:=\left\langle f_{i, j}: i=1, \ldots, m, j=1, \ldots, k\right\rangle .
$$

We also define the approximate multiplication matrices of the radical of $C_{1} \cup \cdots \cup$ $C_{k}$ with respect to the basis $\left[b_{1}, \ldots, b_{k}\right]$ to be the matrices $M_{x_{1}}^{\prime}, \ldots, M_{x_{m}}^{\prime} \in \mathbb{C}(\varepsilon)^{k \times k}$ where

$$
\left[M_{x_{i}}^{\prime}\right]_{j, s}:=\left[\mathbf{v}_{i, j}\right]_{s} \quad i=1, \ldots, m, j, s=1, \ldots, k .
$$

Remark 7.2. We can also define the approximate multiplication matrices of the radical of $C_{1} \cup \cdots \cup C_{k}$ from a system of multiplication matrices of $C_{1} \cup \cdots \cup C_{k}$ with respect to $B$ by changing the basis as follows: Let $r_{k+1}, \ldots, r_{n} \in \mathbb{C}(\varepsilon)^{n}$ be a basis for the nullspace of the first $k$ rows of $P R$. Let $v_{1}, \ldots, v_{k} \in \mathbb{C}^{n}$ be such that $B^{\prime}:=\left[v_{1}, \ldots, v_{k}, r_{k+1}, \ldots, r_{n}\right]$ forms a basis for $\mathbb{C}(\varepsilon)^{n}$. Let $M_{x_{1}}, \ldots, M_{x_{m}} \in$ $\mathbb{C}(\varepsilon)^{n \times n}$ be the multiplication matrices of the clusters $C_{1} \cup \cdots \cup C_{k}$ with respect to the basis $B^{\prime}$. Then the approximate multiplication matrices of the radical of $C_{1} \cup \cdots \cup C_{k}$ with respect to $\left[v_{1}, \ldots, v_{k}\right]$ are the matrices $M_{x_{1}}^{\prime}, \ldots, M_{x_{m}}^{\prime} \in \mathbb{C}(\varepsilon)^{k \times k}$ obtained as the principal $k \times k$ submatrices of $M_{x_{1}}, \ldots, M_{x_{m}}$, respectively. Note that the eigenvalues of $M_{x_{i}}$ are the $x_{i}$ coordinates of the elements of the clusters reordered in a way that the first $k$ correspond to one eigenvalue from each cluster. However, we also remark that we have to be careful with the multiplication matrices $M_{x_{1}}, \ldots, M_{x_{m}}$ since they are not always continuous at $\varepsilon=0$, as noted in Remark 5.5, thus we cannot consider their entries as elements of $\mathbb{C}[[\varepsilon]]$. That is the reason we chose to define the approximate radical as in Definition [7.1.

The next proposition asserts that when $\varepsilon=0$ our definition gives the multiplication matrices of the radical ideal.

Proposition 7.3. Using the assumptions of Definition 7.1 the coordinates of the vectors $\mathbf{v}_{i, j} \in \mathbb{C}(\varepsilon)^{k}$ defined in (14) are continuous in $\varepsilon=0$ for all $i=1, \ldots, m$ and $j=1, \ldots, k$. Furthermore, the points $\mathbf{z}_{1}, \ldots, \mathbf{z}_{k}$ are common roots of the polynomials $\left\{\left.f_{i, j}\right|_{\varepsilon=0}: i=1, \ldots, m, j=1, \ldots, k\right\}$, and the matrices

$$
\left.M_{x_{1}}^{\prime}\right|_{\varepsilon=0}, \ldots,\left.M_{x_{m}}^{\prime}\right|_{\varepsilon=0}
$$

form a system of multiplication matrices for the algebra $\mathbb{C}[\mathbf{x}] / \sqrt{I}$.

Proof. Using Assumption 5.4 the continuity of the coordinates of the vectors $\mathbf{v}_{i, j} \in \mathbb{C}(\varepsilon)^{k}$ follows from our assumption that the $k \times k$ principal submatrix $\tilde{R}^{(k)}$ of $\tilde{R}$ is nonsingular at $\varepsilon=0$. 
Next we show that $\mathbf{z}_{1}, \ldots, \mathbf{z}_{k}$ are roots of $\left.f_{i, j}\right|_{\varepsilon=0}$ for all $i \in\{1, \ldots, m\}$ and $j \in\{1, \ldots, k\}$. Fix $i$ and $j$. Assume that

$$
x_{i} b_{j}-\left(\sum_{s=1}^{k} w_{i, j, s} b_{s}\right)=0
$$

is satisfied by $\mathbf{z}_{1}, \ldots, \mathbf{z}_{k}$, which is equivalent to the column vectors

$$
\mathbf{w}_{i, j}:=\left[w_{i, j, 1}, \ldots, w_{i, j, k},-1\right]^{T}
$$

satisfying the homogeneous linear system with coefficient matrix $W$ defined to be the transpose of the $(k+1) \times k$ Vandermonde matrix of $\mathbf{z}_{1}, \ldots, \mathbf{z}_{k}$ with respect to $\left[b_{1}, \ldots, b_{k}, x_{i} b_{j}\right]$.

On the other hand, by (14), the vector $\left[\mathbf{v}_{i, j} \mid-1\right]_{\varepsilon=0}$ is in the nullspace of the $k \times(k+1)$ matrix $\left[\tilde{R}^{(k)} \mid \mathbf{r}_{i j}\right]_{\varepsilon=0}$. We have

$$
\left[\tilde{R}^{(k)} \mid \mathbf{r}_{i j}\right]_{\varepsilon=0}=V_{1} V_{2}^{T}
$$

where $V_{1}$ and $V_{2}$ are the Vandermonde matrices of $C_{1}, \ldots, C_{k}$ at $\varepsilon=0$ corresponding respectively to $\left[b_{\sigma(1)}, \ldots, b_{\sigma(k)}\right]$ and $\left[b_{1}, \ldots, b_{k}, x_{i} b_{j}\right]$, thus $V_{2}^{T}$ is the same as $W$ except the row corresponding to $\mathbf{z}_{s}$ is repeated $n_{s}$ times for $s=1, \ldots, k$. This implies that the nullspace of $W$ is a subset of the nullspace of $\left[\tilde{R}^{(k)} \mid \mathbf{r}_{i j}\right]_{\varepsilon=0}$. But since both nullspaces has dimension one, we must have $\mathbf{w}_{i, j}=\left[\mathbf{v}_{i, j} \mid-1\right]_{\varepsilon=0}$, i.e. $\left.f_{i, j}\right|_{\varepsilon=0}=0$ is satisfied by $\mathbf{z}_{1}, \ldots, \mathbf{z}_{k}$.

Next we prove that the matrices $\left.M_{x_{1}}^{\prime}\right|_{\varepsilon=0}, \ldots,\left.M_{x_{d}}^{\prime}\right|_{\varepsilon=0}$ form a system of multiplication matrices for $\mathbb{C}[\mathbf{x}] / \sqrt{I}$. First note that for any $g \in \mathbb{C}[\mathbf{x}]$, if $\mathbf{z}$ is a common root of the system

$$
g b_{j}-\sum_{s=1}^{k} c_{j, s} b_{s}=0 \quad j=1, \ldots, k
$$

and $\mathbf{z}$ is not a common root of $b_{1}, \ldots, b_{k}$ then $g(\mathbf{z})$ is an eigenvalue of the matrix $M_{g}:=\left[c_{j, s}\right]_{j, s=1}^{s}$ with corresponding eigenvector $\left[b_{1}(\mathbf{z}), \ldots, b_{k}(\mathbf{z})\right]^{T} \neq 0$. Our assumption that $\left.\tilde{R}^{(k)}\right|_{\varepsilon}=0$ has rank $k$ implies that the vectors $\left[b_{1}\left(\mathbf{z}_{s}\right), \ldots, b_{k}\left(\mathbf{z}_{s}\right)\right]^{T}$ for $s=1, \ldots, k$ are linearly independent, thus they form a common eigensystem for the matrices $\left.M_{x_{1}}^{\prime}\right|_{\varepsilon=0}, \ldots,\left.M_{x_{d}}^{\prime}\right|_{\varepsilon=0}$. Thus, they pairwise commute and their eigenvalues are the coordinates of $\mathbf{z}_{1}, \ldots, \mathbf{z}_{k}$, proving the claim.

Remark 7.4. Without further assumptions on the polynomials $b_{1}, \ldots, b_{k}$ we cannot guarantee that the polynomials $\left.f_{i, j}\right|_{\varepsilon=0}$ have no roots outside of $\mathbf{z}_{1}, \ldots, \mathbf{z}_{k}$. For example, if $k=d=1$ and $\mathbf{z}_{1}=c \neq 0$ but $b_{1}=x$, then $f_{11}=x^{2}-c x$ which also have 0 as a root. However, if we assume that $b_{1}, \ldots, b_{k}$ have no common roots in $\mathbb{C}^{m}$ (e.g. $\left.1 \in\left\{b_{1}, \ldots, b_{k}\right\}\right)$ then all common roots of the polynomials $\left.f_{i, j}\right|_{\varepsilon=0}$ correspond to eigenvalues and eigenvectors of $\left.M_{x_{i}}^{\prime}\right|_{\varepsilon=0}$. Since $\mathbf{z}_{\mathbf{1}}, \ldots, \mathbf{z}_{\mathbf{k}}$ already provides a full system of eigenvectors for $\left.M_{x_{i}}^{\prime}\right|_{\varepsilon=0}$, the polynomials $\left.f_{i, j}\right|_{\varepsilon=0}$ cannot have any other distinct root. 
Our last result gives an asymptotic description of the roots of the polynomials $\left\{f_{i j}\right\}$ in the case when $\varepsilon \neq 0$. Since the coordinates of the vectors $\mathbf{v}_{i, j}$ are continuous in $\varepsilon=0$ we can take their Taylor expansion around $\varepsilon=0$ and consider them as elements of the formal series ring $\mathbb{C}[[\varepsilon]]$, as described in Definition $[5.3$ In this setting we will show that the roots of the system $\left\{f_{i j}\right\}$ are the centers of gravity (or arithmetic means) of the clusters, modulo $\varepsilon^{2}$. Since the arithmetic mean of a cluster is known to be better conditioned than the individual roots in the clusters (c.f. [30, 10]), our result is therefore stable for small enough values of $\varepsilon$.

Proposition 7.5. Let $B=\left[b_{1}, \ldots, b_{n}\right],\left\{\mathbf{z}_{1}, \ldots, \mathbf{z}_{k}\right\}$ and for $i=1, \ldots, k$

$$
\begin{aligned}
C_{i}= & \left\{z_{i, 1}+\delta_{i, 1,1} \varepsilon, \ldots, z_{i, m}+\delta_{i, 1, m} \varepsilon\right], \ldots, \\
& \left.\ldots\left[z_{i, 1}+\delta_{i, n_{i}, 1} \varepsilon, \ldots, z_{i, m}+\delta_{i, n_{i}, m} \varepsilon\right]\right\}
\end{aligned}
$$

be as in Definition 5.3. Let $\vec{\xi}_{s}=\left[\xi_{s, 1}, \ldots, \xi_{s, m}\right]$ for $s=1, \ldots k$ be defined as

$$
\xi_{s, i}:=z_{s, i}+\frac{\sum_{r=1}^{n_{s}} \delta_{s, r, i}}{n_{s}} \varepsilon \quad i=1, \ldots, m .
$$

Then $\vec{\xi}_{1}, \ldots, \vec{\xi}_{k}$ satisfy modulo $\varepsilon^{2}$ the defining equations $\left\{f_{i, j}\right\}$ of the approximate radical ideal of $C_{1} \cup \cdots \cup C_{k}$ defined in Definition 7.1.

Proof. Fix $i \in\{1, \ldots, m\}$ and $j \in\{1, \ldots, k\}$. Define $W$ to be the transpose of the $(k+1) \times k$ Vandermonde matrix of $\vec{\xi}_{1}, \ldots, \vec{\xi}_{k}$ with respect to $\left[b_{1}, \ldots, b_{k}, x_{i} b_{j}\right]$, i.e.

$$
W:=\left[b_{t}\left(\vec{\xi}_{s}\right) \mid\left(x_{i} b_{j}\right)\left(\vec{\xi}_{s}\right)\right]_{s, t=1}^{k} .
$$

Also define $S$ to be the $(k+1) \times k$ augmented matrix

$$
S:=\left[\tilde{R}^{(k)} \mid \mathbf{r}_{i, j}\right]
$$

where $\tilde{R}^{(k)}$ and $\mathbf{r}_{i, j}$ was defined in Definition 7.1 . Assume that

$$
x_{i} b_{j}-\left(\sum_{s=1}^{k} w_{i, j, s} b_{s}\right) \equiv 0 \bmod \varepsilon^{2}
$$

is satisfied by $\vec{\xi}_{s}=\left[\xi_{s, 1}, \ldots, \xi_{s, m}\right]$ for $s=1, \ldots, k$, which is equivalent for the column vector

$$
\mathbf{w}_{i, j}:=\left[w_{i, j, 1}, \ldots, w_{i, j, k},-1\right]^{T}
$$

to satisfy the homogeneous linear system with coefficient matrix $W$ modulo $\varepsilon^{2}$. On the other hand, from the definition of the approximate radical ideal in Definition 7.1, we also have that the augmented vector $\left[\mathbf{v}_{i, j} \mid-1\right]$ is a solution of the homogeneous system corresponding to $S$. By our assumption that $\operatorname{det}\left(\left.\tilde{R}^{(k)}\right|_{\varepsilon=0}\right) \neq 0$, we also have that $\operatorname{det}\left(\tilde{R}^{(k)}\right) \not \equiv 0 \bmod \varepsilon^{2}$, which implies that both $S$ and $W$ have nullspace of dimension 1 modulo $\varepsilon^{2}$. Thus it is enough to show that $\mathbf{w}_{i, j}$ is in the nullspace of $S$ modulo $\varepsilon^{2}$, that will imply that $\mathbf{w}_{i, j} \equiv\left[\mathbf{v}_{i, j} \mid-1\right] \bmod \varepsilon^{2}$. 
Write

$$
\mathbf{w}_{i, j} \equiv \mathbf{w}_{i, j}^{(0)}+\mathbf{w}_{i, j}^{(1)} \varepsilon \quad W \equiv W^{(0)}+W^{(1)} \varepsilon \quad S \equiv S^{(0)}+S^{(1)} \varepsilon \quad \bmod \varepsilon^{2} .
$$

At $\varepsilon=0$ we showed in the proof of Proposition 7.3 that if $\mathbf{w}_{i, j}^{(0)}$ is in the nullspace of $W^{(0)}$ then it is also in the nullspace of $S^{(0)}$.

It remains to prove that $W^{(1)} \mathbf{w}_{i, j}^{(0)}+W^{(0)} \mathbf{w}_{i, j}^{(1)}=0$ implies $S^{(1)} \mathbf{w}_{i, j}^{(0)}+S^{(0)} \mathbf{w}_{i, j}^{(1)}=$ 0 . We use the fact that

$$
S^{(0)}=V_{1} V_{2}^{T} \text { and } S^{(1)}=\bar{V}_{1} W^{(1)}+\left(W_{1}^{(1)}\right)^{T} \bar{V}_{2}^{T}
$$

where $V_{1}$ and $V_{2}$ are the Vandermonde matrices of $C_{1} \cup \cdots \cup C_{k}$ at $\varepsilon=0$ corresponding respectively to $\left[b_{\sigma(1)}, \ldots, b_{\sigma(k)}\right]$ and $\left[b_{1}, \ldots, b_{k}, x_{i} b_{j}\right], W_{1}$ is the Vandermonde matrix corresponding to $\vec{\xi}_{1}, \ldots, \vec{\xi}_{k}$ with respect to $\left(b_{\sigma(1)}, \ldots, b_{\sigma(k)}\right)$, and $\bar{V}_{1}$ and $\bar{V}_{2}$ are the same as $V_{1}$ and $V_{2}$, except the row corresponding to $\mathbf{z}_{s}$ appears only once and it is multiplied by $n_{s}$. Since $\mathbf{w}_{i, j}^{(0)}$ is in the nullspace of $W^{(0)}$, it is also in the nullspace of $\bar{V}_{2}^{T}$, thus it remains to prove that

$$
\bar{V}_{1} W^{(1)} \mathbf{w}_{i, j}^{(0)}+V_{1} V_{2}^{T} \mathbf{w}_{i, j}^{(1)}=0
$$

Since $W^{(1)} \mathbf{w}_{i, j}^{(0)}=-W^{(0)} \mathbf{w}_{i, j}^{(1)}$ by assumption, (22) is equivalent to

$$
\left[-\bar{V}_{1} W^{(0)}+V_{1} V_{2}^{T}\right] \mathbf{w}_{i, j}^{(1)}=0 .
$$

But it is easy to see that $\bar{V}_{1} W^{(0)}=V_{1} V_{2}^{T}$, which proves the claim.

As a corollary of the previous proposition we get that modulo $\varepsilon^{2}$ the approximate multiplication matrices $M_{x_{1}}^{\prime}, \ldots, M_{x_{d}}^{\prime}$ form a pairwise commuting system of multiplication matrices for the roots $\vec{\xi}_{1}, \ldots, \vec{\xi}_{k}$.

Corollary 7.6. Using the notation of Definition 7.1 and Proposition 7.5 we have that for all $i=1, \ldots, k$ and $j=1, \ldots, d$

$$
M_{x_{j}}^{\prime} \mathbf{e}_{\vec{\xi}_{i}} \equiv \xi_{i, j} \mathbf{e}_{\vec{\xi}_{i}} \bmod \varepsilon^{2}
$$

where

$$
\mathbf{e}_{\vec{\xi}_{i}}:=\left[b_{s}\left(\vec{\xi}_{i}\right)\right]_{s=1}^{k} .
$$

Thus the vectors $\left\{\mathbf{e}_{\vec{\xi}_{i}}\right\}_{i=1}^{k}$ form a common eigensystem for the approximate multiplication matrices $M_{x_{1}}^{\prime}, \ldots, M_{x_{d}}^{\prime}$ modulo $\varepsilon^{2}$, which also implies that they are pairwise commuting modulo $\varepsilon^{2}$, i.e. the entries of the commutators $M_{x_{i}}^{\prime} M_{x_{j}}^{\prime}-M_{x_{j}}^{\prime} M_{x_{i}}^{\prime}$ are all divisible by $\varepsilon^{2}$.

Remark 7.7. In practice, for any particular choice of $\varepsilon \in \mathbb{R}_{+}$the system $\left\{f_{i, j}\right\}$ is not necessary consistent. Also, the approximate multiplication matrices $M_{x_{1}}^{\prime}, \ldots, M_{x_{m}}^{\prime}$ are not pairwise commuting, and therefore not simultaneously diagonalizable. However, one can take any consistent subsystem of $\left\{f_{i, j}\right\}$ such that it defines each 
of the coordinates and solve this subsystem in order to obtain the solutions. Another approach is the one described in [30, 10]: If the distance of the clusters from each other were order of magnitude larger than the size of the clusters then a random linear combination of the matrices $M_{x_{1}}^{\prime}, \ldots, M_{x_{d}}^{\prime}$ will have all its eigenvalues distinct with high probablility. Using the eigensystem of this random combination one can approximately diagonalize all of the approximate multiplication matrices $M_{x_{1}}^{\prime}, \ldots, M_{x_{d}}^{\prime}$. Then by Corollary 7.6 and [10, Proposition 8] the entries outside of the diagonal of the resulting matrices will be small, asymptotically $\varepsilon^{2}$. Taking the $i$-th diagonal entry of these nearly diagonal matrices will give the coordinates of the $i$-th root of the approximate radical, which by Proposition 7.5 is approximately the arithmetic mean of a cluster.

Example 7.8. Our last example is similar to Example 3.3 but here we increased the size of the clusters. Consider the polynomial system given by

$$
\begin{aligned}
\tilde{f}_{1}=x_{1}^{2} & +3.99980 x_{1} x_{2}-5.89970 x_{1}+3.81765 x_{2}^{2}-11.25296 x_{2} \\
& +8.33521 \\
\tilde{f}_{2}=x_{1}^{3} & +12.68721 x_{1}^{2} x_{2}-2.36353 x_{1}^{2}+81.54846 x_{1} x_{2}^{2}-177.31082 x_{1} x_{2} \\
& +73.43867 x_{1}-x_{2}^{3}+6 x_{2}^{2}+x_{2}+5 \\
\tilde{f}_{3}=x_{1}^{3} & +8.04041 x_{1}^{2} x_{2}-2.16167 x_{1}^{2}+48.83937 x_{1} x_{2}^{2}-106.72022 x_{1} x_{2} \\
& +44.00210 x_{1}-x_{2}^{3}+4 x_{2}^{2}+x_{2}+3
\end{aligned}
$$

which has a cluster of three common roots, $[0.8999,1],[1,1],[1,0.8999]$ around $[1,1]$ and a cluster of two common roots, $[-1,2],[-1.0999,2]$ around $[-1,2]$. The clusters has size at most $\varepsilon=0.1$. Using Chardin's subresultant method, we obtained the multiplication matrices for this system, with respect to the basis $B=$ $\left[1, x_{1}, x_{2}, x_{1} x_{2}, x_{1}^{2}\right]$ and computed the matrix of traces associated to the system, which is

$$
R=\left[\begin{array}{rrrrr}
5 & 0.79999 & 6.89990 & -1.40000 & 5.01960 \\
0.79999 & 5.01960 & -1.40000 & 7.12928 & 0.39812 \\
6.89990 & -1.40000 & 10.80982 & -5.68988 & 7.12928 \\
-1.40000 & 7.12928 & -5.68988 & 11.45876 & -2.03262 \\
5.01960 & 0.39812 & 7.12928 & -2.03262 & 5.11937
\end{array}\right]
$$

After 2 steps of GECP on the matrix of traces we find the partially reduced matrix $U_{2}$ :

$$
U_{2}=\left[\begin{array}{rrrrr}
11.45876 & -5.68988 & 7.12928 & -1.40000 & -2.03262 \\
0 & 7.98449 & 2.14006 & 6.20472 & 6.11998 \\
0 & 0 & 0.01039 & 0.00799 & 0.02243 \\
0 & 0 & 0.00799 & 0.00728 & 0.01544 \\
0 & 0 & 0.02243 & 0.01544 & 0.06796
\end{array}\right]
$$

with columns permuted to correspond to the basis $\left[x_{1} x_{2}, x_{2}, x_{1}, 1, x_{1}^{2}\right]$.

We also computed the singular values of $R$ :

$$
\text { [24.06746, 13.29215, 0.04397, 0.00362, 0.00035]. }
$$

We indeed have that the entries in the last three rows of $U_{2}$ and the third singular value $\sigma_{3}$ are of the order of $\varepsilon^{2}$, which would determine the numerical rank of $R$ to be 2. 
By considering its last three rows of $U_{2}$ as zero, we compute the nullspace of the resulting matrix, which gives the following generators of $\sqrt{I} / \tilde{I}$,

$$
\begin{aligned}
& r_{3}=x_{2}-1.46302+0.510803 x_{1}, \\
& r_{4}=x_{1} x_{2}+0.51920-1.505323 x_{1}, \\
& r_{5}=x_{1}^{2}-1.01587+0.08562 x_{1} .
\end{aligned}
$$

From these we can define the multiplication matrices for $x_{1}$ and $x_{2}$ in $\mathbb{C}[\mathbf{x}] / \widetilde{\sqrt{I}}$ in the basis $\left[1, x_{1}\right]$ :

$$
\mathcal{M}_{x_{1}}^{\prime}=\left[\begin{array}{cc}
0 & 1 \\
1.01587 & -0.08562
\end{array}\right] \quad \mathcal{M}_{x_{2}}^{\prime}=\left[\begin{array}{cc}
1.46302 & -0.51080 \\
-0.51920 & 1.50533
\end{array}\right]
$$

These matrices do not commute but their commutator have small entries:

$$
\mathcal{M}_{x_{1}}^{\prime} \mathcal{M}_{x_{2}}^{\prime}-\mathcal{M}_{x_{2}}^{\prime} \mathcal{M}_{x_{1}}^{\prime}=\left[\begin{array}{cc}
-0.000293 & -0.00143 \\
0.00147 & 0.000293
\end{array}\right] .
$$

Thus the multiplication matrices are "almost" simultaneously diagonalizable. Following the method in [10, we get the following approximate diagonalizations of $\mathcal{M}_{x_{1}}^{\prime}$ and $\mathcal{M}_{x_{2}}^{\prime}$ using the eigenspace of $\mathcal{M}_{x_{1}}^{\prime}+\mathcal{M}_{x_{2}}^{\prime}$ :

$$
\mathcal{M}_{x_{1}}^{\prime} \sim\left[\begin{array}{cc}
-1.05162 & 0.001765 \\
0.00116 & 0.966001
\end{array}\right] \quad \mathcal{M}_{x_{2}}^{\prime} \sim\left[\begin{array}{cc}
1.99959 & -0.001768 \\
-0.001169 & 0.968759
\end{array}\right] \text {. }
$$

The corresponding diagonal entries give the solutions [-1.05162,1.99959] and $[0.966001,0.968759]$ which are within 0.00167 distance from the centers of gravity of the clusters in the $\infty$-norm.

\section{References}

1. Inés Armendáriz and Pablo Solernó, On the computation of the radical of polynomial complete intersection ideals, AAECC-11: Proceedings of the 11th International Symposium on Applied Algebra, Algebraic Algorithms and Error-Correcting Codes (London, UK), Springer-Verlag, 1995, pp. 106-119.

2. Z. Bai, J. Demmel, and A. McKenney, On the conditioning of the nonsymmetric eigenproblem: Theory and software, Tech. report, Knoxville, TN, USA, 1989.

3. E. Becker and T. Wörmann, Radical computations of zero-dimensional ideals and real root counting, Selected papers presented at the international IMACS symposium on Symbolic computation, new trends and developments (Amsterdam, The Netherlands, The Netherlands), Elsevier Science Publishers B. V., 1996, pp. 561-569.

4. Emmanuel Briand and Laureano Gonzalez-Vega, Multivariate Newton sums: Identities and generating functions, Communications in Algebra 30 (2001), no. 9, 45274547 .

5. J.P. Cardinal and B. Mourrain, Algebraic approach of residues and applications, Proceedings of AMS-Siam Summer Seminar on Math. of Numerical Analysis (Park City, Utah, 1995) (J. Reneger, M. Shub, and S. Smale, eds.), Lectures in Applied Mathematics, vol. 32, 1996, pp. 189-219.

6. E. Cattani, A. Dickenstein, and B. Sturmfels, Computing multidimensional residues, Algorithms in algebraic geometry and applications (Santander, 1994), Progr. Math. vol. 143, Birkhäuser, Basel, 1996, pp. 135-164. 
7. Eduardo Cattani, Alicia Dickenstein, and Bernd Sturmfels, Residues and resultants, J. Math. Sci. Univ. Tokyo 5 (1998), no. 1, 119-148.

8. Marc Chardin, Multivariate subresultants, Journal of Pure and Applied Algebra 101 (1995), 129-138.

9. Robert M. Corless, Gröbner bases and matrix eigenproblems, ACM SIGSAM Bulletin 30 (1996), no. 4, 26-32.

10. Robert M. Corless, Patrizia M. Gianni, and Barry M. Trager, A reordered Schur factorization method for zero-dimensional polynomial systems with multiple roots, ISSAC '97, 1997, pp. 133-140.

11. Robert M. Corless, Patrizia M. Gianni, Barry M. Trager, and Stephen M. Watt, The singular value decomposition for polynomial systems, ISSAC '95, 1995, pp. 195-207.

12. David A. Cox, John B. Little, and Don O'Shea, Using algebraic geometry, Graduate Texts in Mathematics, vol. 185, Springer-Verlag, NY, 1998, 499 pages.

13. Carlos D'Andrea and Gabriela Jeronimo, Rational formulas for traces in zerodimensional algebras., http://arxiv.org/abs/math.AC/0503721 (2005).

14. Barry H. Dayton and Zhonggang Zeng, Computing the multiplicity structure in solving polynomial systems, ISSAC '05, 2005, pp. 116-123.

15. James Demmel, Accurate singular value decompositions of structured matrices, SIMAX (1999).

16. James Demmel and Plamen Koev, Accurate SVD's of polynomial vandermonde matrices involving orthonormal polynomials, Linear Algebra Applications, to appear (2005).

17. Gema M. Díaz-Toca and Laureano González-Vega, An explicit description for the triangular decomposition of a zero-dimensional ideal through trace computations, Symbolic computation: solving equations in algebra, geometry, and engineering (South Hadley, MA, 2000), Contemp. Math., vol. 286, AMS, 2001, pp. 21-35.

18. L.E. Dickson, Algebras and Their Arithmetics, University of Chicago Press, 1923.

19. Katalin Friedl and Lajos Rónyai, Polynomial time solutions of some problems of computational algebra, STOC '85, ACM Press, 1985, pp. 153-162.

20. Gene H. Golub and Charles F. Van Loan, Matrix computations, third ed., Johns Hopkins Studies in the Mathematical Sciences, Johns Hopkins University Press, Baltimore, MD, 1996.

21. V. Hribernig and H. J. Stetter, Detection and validation of clusters of polynomial zeros, J. Symb. Comput. 24 (1997), no. 6, 667-681.

22. Itnuit Janovitz-Freireich, Lajos Rónyai, and Ágnes Szántó, Approximate radical of ideals with clusters of roots, ISSAC '06: Proceedings of the 2006 international symposium on Symbolic and algebraic computation (New York, NY, USA), ACM Press, 2006, pp. 146-153.

23. W. Kahan, Numerical linear algebra, Canadian Mathematical Bulletin (1966), no. 9, 757-801.

24. E. Kaltofen, On computing determinants of matrices without divisions, ISSAC'92 (New York, N. Y.) (P. S. Wang, ed.), ACM Press, 1992, pp. 342-349.

25. Erich Kaltofen and John May, On approximate irreducibility of polynomials in several variables, ISSAC '03, 2003, pp. 161-168. 
26. K. H. Ko, T. Sakkalis, and N. M. Patrikalakis, Nonlinear Polynomial Systems: Multiple Roots and their Multiplicities, Proceedings of the Shape Modeling International 2004 (2004).

27. Daniel Lazard, Resolution des systemes d'equations algebriques, Theoret. Comp. Sci. 15 (1981), no. 1, French, English summary.

28. G. Lecerf, Quadratic Newton iterarion for systems with multiplicity, Foundations of Computational Mathematics (2002), no. 2, 247-293.

29. Anton Leykin, Jan Verschelde, and Ailing Zhao, Evaluation of Jacobian matrices for Newton's method with deflation to approximate isolated singular solutions of polynomial systems, SNC 2005 Proceedings. International Workshop on Symbolic-Numeric Computation. (Dongming Wang and Lihong Zhi, eds.), 2005, pp. 19-28.

30. Dinesh Manocha and James Demmel, Algorithms for Intersecting Parametric and Algebraic Curves II: Multiple Intersections, Graphical Models and Image Processing 57 (1995), no. 2, 81-100.

31. Maria Grazia Marinari, Teo Mora, and Hans Michael Möller, Gröbner duality and multiplicities in polynomial system solving, ISSAC '95, 1995, pp. 167-179.

32. H. Michael Möller and Hans J. Stetter, Multivariate polynomial equations with multiple zeros solved by matrix eigenproblems, Numerische Matematik 70 (1995), 311-329.

33. Alexander P. Morgan, Andrew J. Sommese, and Charles W. Wampler, Computing singular solutions to nonlinear analytic systems., Numer. Math. 58 (1991), no. 7, 669-684.

34. _ Computing singular solutions to polynomial systems, Adv. Appl. Math. 13 (1992), no. 3, 305-327.

35. - A power series method for computing singular solutions to nonlinear analytic systems, Numer. Math. 63 (1992), no. 3, 391-409.

36. Schuichi Moritsugu and Kazuko Kuriyama, A linear algebra method for solving systems of algebraic equations, RISC-Linz Report Series, vol. 35, 1997.

37. On multiple zeros of systems of algebraic equations, ISSAC '99, 1999, pp. 2330.

38. Bernard Mourrain, Generalized normal forms and polynomial system solving, ISSAC '05, 2005, pp. 253-260.

39. Takeo Ojika, Modified deflation algorithm for the solution of singular problems. I. A system of nonlinear algebraic equations, J. Math. Anal. Appl. 123 (1987), no. 1, $199-221$.

40. __ Modified deflation algorithm for the solution of singular problems. II. Nonlinear multipoint boundary value problems, J. Math. Anal. Appl. 123 (1987), no. 1, 222-237.

41. Takeo Ojika, Satoshi Watanabe, and Taketomo Mitsui, Deflation algorithm for the multiple roots of a system of nonlinear equations, J. Math. Anal. Appl. 96 (1983), no. 2, 463-479.

42. Richard S. Pierce, Associative algebras, Graduate Text in Mathematics, vol. 88, Springer-Verlag, 1982. 
43. Fabrice Rouiller., Solving zero-dimensional systems through the rational univariate representation., AAECC: Applicable Algebra in Engineering, Communication and Computing, vol. 9, Springer Berlin / Heidelberg, 1999, pp. 433-461.

44. Tateaki Sasaki and Matu-Tarow Noda, Approximate square-free decomposition and root-finding of ill-conditioned algebraic equations, J. Inform. Process. 12 (1989), no. 2, 159-168.

45. Éric Schost, Personal communication, 2005.

46. Victor Shoup, Efficient computation of minimal polynomials in algebraic extensions of finite fields, ISSAC '99, 1999, pp. 53-58.

47. Hans J. Stetter, Analysis of zero clusters in multivariate polynomial systems, ISSAC '96, 1996, pp. 127-136.

48. __ Numerical polynomial algebra, Society for Industrial and Applied Mathematics, 2004.

49. Agnes Szanto, Solving over-determined systems by subresultant methods, Preprint, 2001.

50. Kazuhiro Yokoyama, Masayuki Noro, and Taku Takeshima, Solutions of systems of algebraic equations and linear maps on residue class rings, J. Symb. Comput. 14 (1992), no. 4, 399-417.

51. Zhonggang Zeng, A method computing multiple roots of inexact polynomials, ISSAC '03, 2003, pp. 266-272.

Itnuit Janovitz-Freireich

Mathematics Department, North Carolina State University, Raleigh, NC, USA.

e-mail: ijanovi2@ncsu.edu

Lajos Rónyai

Computer and Automation Institute, Hungarian Academy of Sciences and Budapest University of Technology and Economics, Budapest, Hungary.

e-mail: lajos@csillag.ilab.sztaki.hu

Ágnes Szántó

Mathematics Department, North Carolina State University, Raleigh, NC, USA.

e-mail: aszanto@ncsu.edu 